\title{
Aggregate-Strength Interaction Test Suite Prioritization
}

\author{
Rubing Huang ${ }^{\mathrm{a}, \mathrm{b}, *}$, Jinfu Chen ${ }^{\mathrm{a}}$, Dave Towey ${ }^{\mathrm{c}}$, Alvin T. S. Chan ${ }^{\mathrm{d}}$, Yansheng Lu ${ }^{\mathrm{b}}$ \\ ${ }^{a}$ School of Computer Science and Telecommunication Engineering, Jiangsu University, Zhenjiang, Jiangsu 212013, P.R. China \\ ${ }^{b}$ School of Computer Science and Technology, Huazhong University of Science and Technology, Wuhan, Hubei 430074, P.R. China \\ ${ }^{c}$ School of Computer Science, The University of Nottingham Ningbo China, Ningbo, Zhejiang 315100, P.R. China. \\ ${ }^{d}$ Department of Computing, The Hong Kong Polytechnic University, Hong Kong, P.R. China
}

\begin{abstract}
Combinatorial interaction testing is a widely used approach. In testing, it is often assumed that all combinatorial test cases have equal fault detection capability, however it has been shown that the execution order of an interaction test suite's test cases may be critical, especially when the testing resources are limited. To improve testing cost-effectiveness, test cases in the interaction test suite can be prioritized, and one of the best-known categories of prioritization approaches is based on "fixed-strength prioritization", which prioritizes an interaction test suite by choosing new test cases which have the highest uncovered interaction coverage at a fixed strength (level of interaction among parameters). A drawback of these approaches, however, is that, when selecting each test case, they only consider a fixed strength, not multiple strengths. To overcome this, we propose a new "aggregate-strength prioritization", to combine interaction coverage at different strengths. Experimental results show that in most cases our method performs better than the test-case-generation, reverse test-case-generation, and random prioritization techniques. The method also usually outperforms "fixed-strength prioritization", while maintaining a similar time cost.
\end{abstract}

Keywords:

Software testing, combinatorial interaction testing, test case prioritization, interaction coverage, fixed-strength prioritization, aggregate-strength prioritization, algorithm

\section{Introduction}

Combinatorial interaction testing [29], is a black-box ${ }_{22}^{21}$ testing method that has been well researched, and applied in ${ }_{23}$ the testing of practical systems [14, 24, 42]. It focuses on ${ }_{24}$ constructing an effective test suite (called an interaction test ${ }_{25}$ suite) in order to catch failures triggered by the interactions ${ }_{26}$ among $k$ parameters of the software under test (SUT). Here, ${ }_{27}$ parameters may represent any factors that affect the running of ${ }_{28}^{27}$ the SUT, such as user inputs, configuration options, etc., and ${ }_{29}$ each parameter may have several valid values. In fact, combinatorial interaction testing provides a trade-off between ${ }_{31}$ testing effectiveness and efficiency, because it only requires coverage of certain key combinations, rather than of all ${ }_{33}$ possible combinations, of parametric values. For instance, ${ }_{34}$ $\tau$-wise $(1 \leq \tau \leq k)$ combinatorial interaction testing, where $\tau$ is ${ }_{35}$ referred to as the level of interaction among parameters ${ }_{36}$ (named strength), constructs an interaction test suite to cover ${ }_{37}$ all possible $\tau$-tuples of parameter values (referred to as $\tau$-wise ${ }_{38}$ parameter value combinations).

*Corresponding author at: School of Computer Science and ${ }^{4}$ Telecommunication Engineering, Jiangsu University, 301 Xuefu Road, ${ }^{42}$ Zhenjiang, Jiangsu 212013, P.R. China.

Email addresses: rbhuang@mail.ujs.edu.cn, or

rbhuang@hust .edu. cn (Rubing Huang), jinfuchen@ujs.edu.cn (Jinfu

Chen), dave.towey@nottingham.edu.cn (Dave Towey),

cstschan@comp. polyu.edu.hk (Alvin T. S. Chan), lys@hust.edu.cn

(Yansheng Lu)
Due to limited testing resources in practical applications where combinatorial interaction testing is used, for example in combinatorial interaction regression testing [32], the execution order of combinatorial test cases can be critical, and therefore the potentially failure-revealing test cases in an interaction test suite should be executed as early as possible. In other words, a well-ordered test case execution may be able to detect failures earlier, and thus enable earlier fault characterization, diagnosis and correction [29]. To improve testing efficiency, interaction test suites can be prioritized [29].

The prioritization of interaction test suites has been well studied [1, 2, 4-7, 18, 30-33, 37, 39, 40], with many techniques having been proposed, such as random prioritization [1] and branch-coverage-based prioritization [32]. A well-studied category of prioritization approaches for interaction test suites is "fixed-strength prioritization", which prioritizes the interaction test suite by repeatedly choosing an unexecuted test case from candidates such that it covers the largest number of uncovered parameter value combinations at a fixed strength [1, 2, 4-7, 18, 30-33, 37, 39, 40]. However, when selecting each unexecuted test case, this strategy only considers interaction coverage of a fixed strength $\tau$, rather than interaction coverage of multiple strengths: Although it focuses on $\tau$-wise interaction coverage, it may neglect $\lambda$-wise $(1 \leq \lambda<\tau)^{1}$ interaction coverage when choosing the next test

${ }^{1}$ For ease of description, in this paper we assume $\tau$ is a constant, because $\tau$ is obtained from an interaction test suite; while $\lambda$ is a variable where $1 \leq \lambda \leq \tau$. 
case. Consequently, "fixed-strength prioritization" may not use sufficient information to guide the prioritization of the interaction test suite - an example of this will be given in the following section.

To evaluate the difference between a combinatorial test case and the already executed test cases, we propose a new dissimilarity measure which considers different interaction coverage at different strengths. Based on this, we present $\mathrm{a}_{52}$ heuristic algorithm which, given an interaction test suite $T$ of ${ }_{53}$ strength $\tau$, chooses an element from among candidates after ${ }_{54}$ comprehensively considering different interaction coverage at ${ }_{55}$ strengths from 1 to $\tau$, and assigning each interaction coverage a weight. The method gives a priority of all strengths from 1 to ${ }^{56}$ $\tau$, and balances $\lambda$-wise interaction coverage for $\lambda=1,2, \ldots, \tau .{ }^{57}$ This proposed method has the advantage over existing 58 prioritization methods by employing more information to guide the prioritization process. We refer to this method as ${ }^{59}$ "aggregate-strength prioritization".

In terms of the rates of covering parameter value ${ }_{61}$ combinations and fault detection, experimental results show ${ }_{62}$ that in most cases our method performs better than the ${ }_{63}$ test-case-generation, reverse test-case-generation, and random prioritizations; and also has better performance than the 64 "fixed-strength prioritization," while maintaining a similar 65 time cost.

This paper is organized as follows: Section 2 introduces ${ }^{67}$ some background information, including combinatorial 68 interaction testing, and test case prioritization. Section 369 describes some related work. Section 4 introduces a 70 motivating example, and then proposes a new prioritization strategy, with an analysis of its properties and time complexity. ${ }^{7}$ Section 5 presents some simulations and experiments with ${ }^{72}$ real-life programs related to the use of the proposed strategy, ${ }^{73}$ and finally, Section 6 presents the conclusions and discusses ${ }^{74}$ potential future work.

\section{Background}

In this section, some fundamental aspects of combinatorial 79 interaction testing and test case prioritization are presented.

\subsection{Combinatorial interaction testing}

Combinatorial interaction testing is used to generate a test ${ }^{83}$ suite to detect faults triggered by interactions among parameters in the SUT. For convenience, in the remainder of this paper we will refer to a combination of parameters as a parameter combination, and a combination of parametric values or a parameter value combination as a value combination.

Definition 1. A test profile, denoted as $T P\left(k,\left|V_{1}\right|\left|V_{2}\right| \cdots\left|V_{k}\right|, \mathscr{D}\right)$, has information about a combinatorial test space of the SUT, including $k$ parameters, $\left|V_{i}\right|(i=1,2, \cdots, k)$ values for the $i$-th parameter, and constraints $\mathscr{D}$ on value combinations.
Table 1: A Test Profile for a SUT

\begin{tabular}{|c|c|c|c|c|}
\hline Parameter & $p_{1}$ & $p_{2}$ & $p_{3}$ & $p_{4}$ \\
\hline \multirow{3}{*}{ Value } & 0 & 3 & 6 & 8 \\
& 1 & 4 & 7 & 9 \\
& 2 & 5 & - & - \\
\hline
\end{tabular}

Table 1 gives an example of a SUT with $\mathscr{D}=\emptyset$, in which there are four parameters, two of which have two values and another two of which have three values: the test profile can be written as $T P\left(4,2^{2} 3^{2}, \emptyset\right)$.

Definition 2. Given a test profile $T P\left(k,\left|V_{1}\right|\left|V_{2}\right| \cdots\left|V_{k}\right|, \mathscr{D}\right)$, a $k$ tuple $\left(v_{1}, v_{2}, \cdots, v_{k}\right)$ is a combinatorial test case for the SUT, where $v_{i} \in V_{i}(i=1,2, \cdots, k)$.

For example, $(0,3,6,8)$ is a 4-tuple combinatorial test case for the SUT shown in Table 1.

Definition 3. The number of parameters required to trigger a failure is referred to as the failure-triggering fault interaction (FTFI) number.

The combinatorial input domain fault model assumes that failures are caused by parameter interactions. For example, if the SUT shown in Table 1 fails when both $p_{2}$ is set to 5 and $p_{3}$ is set to 6 , this failure is caused by the parameter interaction $\left(p_{2}, p_{3}\right)$, and therefore, the FTFI number is 2 .

In combinatorial interaction testing, a covering array is generally used to represent an interaction test suite.

Definition 4. Given a $T P\left(k,\left|V_{1}\right|\left|V_{2}\right| \cdots\left|V_{k}\right|, \mathscr{D}\right)$, an $N \times k$ matrix is a $\tau$-wise $(1 \leq \tau \leq k)$ covering array, denoted $C A\left(N ; \tau, k,\left|V_{1}\right|\right.$ $\left.\left|V_{2}\right| \cdots\left|V_{k}\right|\right)$, which satisfies the following properties: (1) each column $i(i=1,2, \cdots, k)$ contains only elements from the set $V_{i}$; and (2) the rows of each $N \times \tau$ sub-matrix cover all $\tau$-wise value combinations from the $\tau$ columns at least once.

Table 2 shows an example covering array for the SUT in Table 1 . The covering array, denoted as $C A\left(9 ; 2,4,2^{2} 3^{2}\right)$, only requires a set of nine test cases in order to cover all 2 -wise value combinations.

Each column of a covering array represents a parameter of the SUT, while each row represents a combinatorial test case. Testing with a $\tau$-wise covering array is called $\tau$-wise

Table 2: $C A\left(9 ; 2,4,2^{2} 3^{2}\right)$ for the $T P\left(4,2^{2} 3^{2}, \emptyset\right)$ shown in Table 1

\begin{tabular}{|c|c|c|c|c|}
\hline Test No. & $p_{1}$ & $p_{2}$ & $p_{3}$ & $p_{4}$ \\
\hline$t c_{1}$ & 0 & 3 & 7 & 9 \\
$t c_{2}$ & 0 & 4 & 6 & 8 \\
$t c_{3}$ & 0 & 5 & 7 & 8 \\
$t c_{4}$ & 1 & 3 & 6 & 9 \\
$t c_{5}$ & 1 & 4 & 7 & 8 \\
$t c_{6}$ & 1 & 5 & 6 & 9 \\
$t c_{7}$ & 2 & 3 & 7 & 8 \\
$t c_{8}$ & 2 & 4 & 6 & 9 \\
$t c_{9}$ & 2 & 5 & 6 & 8 \\
\hline
\end{tabular}


combinatorial interaction testing. In this paper, we focus on 46 $\tau$-wise covering arrays, rather than on other interaction test 47 suites such as variable-strength covering array [9].

In $\tau$-wise combinatorial interaction testing, the uncovered 49 $\lambda$-wise value combinations distance $\left(U V C D_{\lambda}\right)$ is a distance (or 50 dissimilarity) measure often used to evaluate combinatorial test 51 cases against an interaction test suite [20].

Definition 5. Given an interaction test suite $T$, strength $\lambda$, and a combinatorial test case $t c$, the uncovered $\lambda$-wise value combinations distance $\left(U V C D_{\lambda}\right)$ of tc against $T$ is defined as:

$$
U V C D_{\lambda}(t c, T)=\left|\operatorname{CombSet}_{\lambda}(t c) \backslash \operatorname{CombSet}_{\lambda}(T)\right|,
$$

where $\mathrm{CombSet}_{\lambda}(\mathrm{tc})$ is the set of all $\lambda$-wise value combinations 56 covered by tc, and $\operatorname{CombSet}_{\lambda}(T)$ is the set covered by all of $T .{ }_{57}$ More specifically, these can be respectively written as follows: 58

$$
\begin{gathered}
\operatorname{CombSet}_{\lambda}(t c)=\left\{\left(v_{j_{1}}, v_{j_{2}}, \cdots, v_{j_{\lambda}}\right) \mid 1 \leq j_{1}<j_{2}<\cdots<j_{\lambda} \leq k\right\} \\
\operatorname{CombSet}_{\lambda}(T)=\bigcup_{t c \in T} \operatorname{CombSet}_{\lambda}(t c)
\end{gathered}
$$

In the past, minimization of the interaction test suite size ${ }^{64}$ has been emphasized in order to achieve the desired coverage, 65 and although the problem of constructing interaction test suites (covering array and variable-strength covering array) is NP-Complete [35], many strategies for building them have been developed, including approaches employing greedy, 66 recursive, heuristic search, and algebraic algorithms and 67 methods (see [29] for more details).

\subsection{Test case prioritization}

Suppose $T=\left\{t c_{1}, t c_{2}, \cdots, t c_{N}\right\}$ is a test suite containing $N^{71}$ test cases, and $S=\left\langle s_{1}, s_{2}, \cdots, s_{N}\right\rangle$ is an ordered set of $T,{ }^{72}$ called a test sequence, where $s_{i} \in T$ and ${ }^{73}$ $s_{i} \neq s_{j}(i, j=1,2, \cdots, N ; i \neq j)$. If $S_{1}=\left\langle s_{1}, s_{2}, \cdots, s_{m}\right\rangle$ and ${ }^{74}$ $S_{2}=\left\langle q_{1}, q_{2}, \cdots, q_{n}\right\rangle$ are two test sequences, we define $\left.S_{1}\right\rangle S_{2}{ }^{75}$ as $\left\langle s_{1}, s_{2}, \cdots, s_{m}, q_{1}, q_{2}, \cdots, q_{n}\right\rangle$; and $T \backslash S$ as the maximal ${ }^{76}$ subset of $T$ whose elements are not in $S$.

Test case prioritization is used to schedule test cases in an ${ }^{78}$ order, so that, according to some criteria (e.g. condition ${ }^{79}$ coverage), test cases with higher priority are executed earlier ${ }^{80}$ in the testing process. A well-prioritized test sequence may ${ }^{81}$ improve the likelihood of detecting faults earlier, which may be especially important when testing with limited test resources. The problem of test case prioritization is defined as follows [34]:

Definition 6. Given $(T, \Omega, g)$, where $T$ is a test suite, $\Omega$ is the ${ }^{84}$ set of all possible test sequences obtained by ordering test cases ${ }^{85}$ of $T$, and $g$ is a function from a given test sequence to an award ${ }^{86}$ value, the problem of test case prioritization is to find an $S \in \Omega_{88}^{87}$ such that:

$$
\left(\forall S^{\prime}\right)\left(S^{\prime} \in \Omega\right)\left(S^{\prime} \neq S\right)\left[g(S) \geq g\left(S^{\prime}\right)\right]
$$

In Eq. (4), $g$ is a function to evaluate a test sequence $S$ by ${ }^{92}$ returning a real number. A well-known function is $a$ weighted ${ }^{93}$ average of the percentage of faults detected (APFD) [13], which is a measure of how quickly a test sequence can detect faults during the execution (that is, the rate of fault detection). Let $T$ be a test suite containing $N$ test cases, and let $F$ be a set of $m$ faults revealed by $T$. Let $S F_{i}$ be the number of test cases in test sequence $S$ of $T$ that are executed until detecting fault $i$. The APFD for test sequence $S$ is given by the following equation from [13]:

$$
A P F D=1-\frac{S F_{1}+S F_{2}+\cdots+S F_{m}}{N \times m}+\frac{1}{2 N} .
$$

The APFD metric, which has been used in practical testing, has a range of $(0,1)$, with higher values implying faster rates of fault detection. Two requirements of the APFD metric are that (1) all test cases in a test sequence should be executed; and (2) all faults should be detected. In practical testing applications, however, it may be that only part of the test sequence is run, and only some of the faults detected. In such cases, the APFD may not be an appropriate evaluation of the fault detection rate. To alleviate the difficulties associated with these two requirements, $\mathrm{Qu}$ et al. [32] have presented a new metric, Normalized APFD (NAPFD), as an enhancement of APFD, and defined it as follows:

$$
N A P F D=p-\frac{S F_{1}+S F_{2}+\cdots+S F_{m}}{N^{\prime} \times m}+\frac{p}{2 N^{\prime}},
$$

where $m$ and $S F_{i}(i=1,2, \cdots, m)$ have the same meaning as in APFD; $p$ represents the ratio of the number of faults identified by selected test cases relative to the number of faults detected by the full test suite; and $N^{\prime}$ is the number of the executed test cases. If a fault $f_{i}$ is never found, then $S F_{i}=0$. If all faults can be detected, and all test cases are executed, NAPFD and APFD are identical, with $p=1.0$ and $N^{\prime}=N$.

Many test case prioritization strategies have been proposed, such as fault severity based prioritization [12], source code based prioritization [34, 41], search based prioritization [26], integer linear programming based prioritization [44], XML-manipulating prioritization [28], risk exposure based prioritization [43], system-level test case prioritization [36], and history based prioritization [21]. Most prioritization strategies can be classified as either meta-heuristic search methods or greedy methods [40].

\section{Related Work}

According to Qu's classification, the prioritization of interaction test suites can generally be divided into two categories: (1) pure prioritization: re-ordering test cases in the interaction test suite; and (2) re-generation prioritization: considering the prioritization principle during the process of interaction test suite generation, that is, generating (or constructing) an interaction test sequence [32]. The method proposed in this paper, as well as the methods used for comparison, belongs to the former category. However, if based on the same prioritization principle, pure prioritization works in a similar manner to re-generation prioritization. For 
example, when testers base prioritization on test case 58 execution time, pure prioritization chooses an element from 59 the given test suite such that it has the lowest execution time, 60 and re-generation prioritization selects (or generates) such 61 elements from the exhaustive test suite (or constructed 62 candidates). In this section, therefore, we do not distinguish 63 between pure and re-generation prioritization.

Bryce and Colbourn [1, 2] initially used four test case 65 weighting distributions to construct interaction test sequences 66 with seeding and constraints, which belongs to the 67 re-generation prioritization category. Bryce et al. [4, 5] 68 proposed a pure prioritization method without considering any 69 other factors (only considering 2-wise and 3-wise interaction 70 coverage) for interaction test suites, and then applied it to the 71 event-driven software. Similarly, Qu et al. [31-33] applied test case weight to the pure prioritization method, and applied this method to configurable systems. They also proposed other test ${ }^{72}$ case weighting distributions based on code coverage [32], specification [32], fault detection [31], and configuration change [31]. Additionally, Chen et al. [7] used an ant colony algorithm to generate prioritized interaction test suites which ${ }_{76}$ considered interaction coverage information.

Srikanth et al. [37] took the cost of installing and building new configurations into consideration for helping prioritize ${ }_{79}$ interaction test suites. Bryce et al. [6] used the length of the test case to represent its cost, and combined it with pair-wise ${ }_{81}$ interaction coverage to guide the prioritization of interaction test suites. Wang et al. [40] combined test case cost with test case weight to prioritize interaction test suites, and also extended this method from lower to higher strengths, and ${ }^{83}$ proposed a series of metrics which have been widely used in ${ }^{84}$ the evaluation of interaction test sequences. Petke et al. [30] ${ }^{85}$ researched the efficiency and fault detection of the pure ${ }^{86}$ prioritization method proposed in $[4,5]$ with other (lower and ${ }^{87}$ higher) strengths. Recently, Huang et al. [18] investigated ${ }^{88}$ adaptive random test case prioritization for interaction test ${ }^{89}$ suites using interaction coverage, a method which, by ${ }^{90}$ replacing the prioritization method in $[4,5]$ attempts to reduce ${ }^{91}$ time costs, while maintaining effectiveness.

Throughout the interaction test suite prioritization process, ${ }^{93}$ the strategies so far mentioned $[1,2,4-7,18,30-33,37,40]^{94}$ do not vary the strength of interaction coverage. For example, ${ }^{95}$ given a strength $\tau$ for prioritization, these prioritization ${ }^{96}$ strategies only consider $\tau$-wise interaction coverage to guide ${ }^{97}$ the test cases selection. These implementations of ${ }^{98}$ "fixed-strength prioritization" are also referred to as ${ }^{99}$ interaction coverage based prioritization (or ICBP). Previous ${ }^{100}$ studies also investigated incremental strengths to prioritize ${ }^{101}$ interaction test suites. For instance, Wang [39] used ${ }^{102}$ incremental strengths, and proposed a pure prioritization ${ }^{103}$ method named inCTPri used to prioritize covering arrays. ${ }^{104}$ More specifically, given a $\tau$-wise covering array ${ }^{105}$ $C A\left(N ; \tau, k,\left|V_{1}\right|\left|V_{2}\right| \cdots\left|V_{k}\right|\right)$, the inCTPri firstly uses interaction ${ }^{100}$ coverage at a low strength (such as $\lambda$ where $1<\lambda \leq \tau$ ) to ${ }^{107}$ prioritize $\mathrm{CA}$; when all $\lambda$-wise value combinations have been ${ }^{108}$ covered by selected test cases, the inCTPri increases $\lambda$ to $\lambda+1$, and then repeats the above process until $\lambda>\tau$. In other words, inCTPri is actually ICBP using different strengths during the prioritization process. Huang et al. [19] proposed two pure prioritization methods for variable-strength covering arrays which exploit the main-strength and sub-strengths of variable-strength covering arrays to guide prioritization.

To date, most interaction test suite prioritization strategies belong to the category of "fixed-strength prioritization", because they only consider a fixed strength when selecting each combinatorial test case from candidates. Few studies have been conducted on the prioritization of interaction test suites using "aggregate-strength prioritization", and our study is, to our best knowledge, the first to use multiple strengths when choosing each combinatorial test case from the candidate elements.

\section{Aggregate-Strength Interaction Test Suite Prioritization}

In this section, we present a motivating example to illustrate the shortcoming of "fixed-strength prioritization", and then introduce a new dissimilarity measure for evaluating combinatorial test cases, the weighted aggregate-strength test case dissimilarity (WASD). We then introduce a heuristic algorithm for prioritizing an interaction test suite based on the WASD measure ("aggregate-strength prioritization" strategy), investigate some of its properties, and give a time complexity analysis.

\subsection{A motivating example}

Given covering array $C A\left(9 ; 2,4,2^{2} 3^{2}\right)$, shown in Table 2, "fixed-strength prioritization" generally uses strength $\tau=2$ to guide the prioritization. More specifically, this strategy chooses the next test case such that it covers the largest number of 2-wise value combinations that have not yet been covered by the already selected test cases (that is, $U V C D_{2}$ ). We assume that "fixed-strength prioritization" is deterministic, e.g. the first candidate is selected as the next test case in situations with more than one best element, and therefore its generated interaction test sequence would be $S_{1}=\left\langle t c_{1}, t c_{2}, t c_{6}, t c_{5}, t c_{7}, t c_{8}, t c_{3}, t c_{4}, t c_{9}\right\rangle . \quad$ Intuitively speaking, $S_{1}$ would face a challenge when a fault $f_{1}$ is triggered by " $P_{1}=2$ ", because it needs to run five test cases $(5 / 9=55.56 \%)$ in order to detect this fault. However, if multiple strengths were used to prioritize this interaction test suite, e.g. strengths 1 and 2, both 1 -wise and 2-wise value combinations would be considered, and therefore we would obtain the interaction test sequence $S_{2}=\left\langle t c_{1}, t c_{9}, t c_{4}, t c_{2}, t c_{5}, t c_{7}, t c_{8}, t c_{3}, t c_{6}\right\rangle . \quad S_{2}$ would only require 2 test cases $(2 / 9=22.22 \%)$ to be run to identify the fault $f_{1}$, which means that $S_{2}$ has a faster fault detection than $S_{1}$.

Motivated by this, it is reasonable to consider different strengths for prioritizing interaction test suites, which may provide better effectiveness (such as fault detection) than "fixed-strength prioritization". 


\subsection{Weighted aggregate-strength test case dissimilarity}

Given an interaction test suite $T$ on ${ }^{27}$ $T P\left(k,\left|V_{1}\right|\left|V_{2}\right| \cdots\left|V_{k}\right|, \mathscr{D}\right)$, a combinatorial test case $t c$, and the ${ }^{28}$ strength $\tau$, the weighted aggregate-strength test case ${ }^{29}$ dissimilarity (WASD) of $t c$ against $T$ is defined as follows:

$$
\operatorname{WASD}(t c, T)=\sum_{\lambda=1}^{\tau}\left(\omega_{\lambda} \times \frac{\left|U V C D_{\lambda}(t c, T)\right|}{C_{k}^{\lambda}}\right),
$$

where $0 \leq \omega_{\lambda} \leq 1.0(\lambda=1,2, \cdots, \tau)$, and $\sum_{\lambda=1}^{\tau} \omega_{\lambda}=1.0$.

Intuitively speaking, $W A S D(t c, T)=0$, if and only if $t c \in T ;{ }^{33}$ $W A S D(t c, T)=1.0$, if and only if any 1 -wise value combination ${ }^{34}$ covered by $t c$ is not covered by $T$. Therefore, the WASD ranges ${ }^{35}$ from 0 to 1.0 .

Here, we present an example to briefly illustrate the WASD. ${ }^{37}$ Considering the combinatorial test cases in Table 2, suppose ${ }^{38}$ interaction test sequence $S=\left\{t c_{1}\right\}$, strength $\tau=2$, two ${ }^{39}$ candidates $t c_{2}$ and $t c_{9}$, and $\omega_{1}=\omega_{2}=\cdots=\omega_{\tau}=1 / \tau$, the WASD of $t c_{2}$ against $T$ is ${ }^{40}$ $W A S D\left(t c_{2}, S\right)=\frac{1}{2} \times \frac{U V C D_{\lambda=1}\left(t c_{2}, S\right)}{C_{4}^{1}}+\frac{1}{2} \times \frac{U V C D_{\lambda=2}\left(t c_{2}, S\right)}{C_{4}^{2}}=41$ $\frac{1}{2} \times \frac{3}{4}+\frac{1}{2} \times \frac{6}{6}=0.375+0.5=0.875$; while the WASD of $t c_{9}{ }^{42}$ against $S \quad$ is $^{43}$ $W A S D\left(t c_{9}, S\right)=\frac{1}{2} \times \frac{U V C D_{\lambda=1}\left(t c_{9}, S\right)}{C_{4}^{1}}+\frac{1}{2} \times \frac{U V C D_{\lambda=2}\left(t c_{9}, S\right)}{C_{4}^{2}}={ }_{44}$ $\frac{1}{2} \times \frac{4}{4}+\frac{1}{2} \times \frac{6}{6}=0.5+0.5=1.0$. In this case, it can be ${ }^{45}$ concluded that the test case $t c_{9}$ would be a better next test case ${ }^{46}$ in $S$ than $t c_{2}$.

\subsection{Algorithm}

In this section, we introduce a new heuristic algorithm, ${ }_{50}^{49}$ namely "aggregate-strength prioritization" strategy (ASPS), to

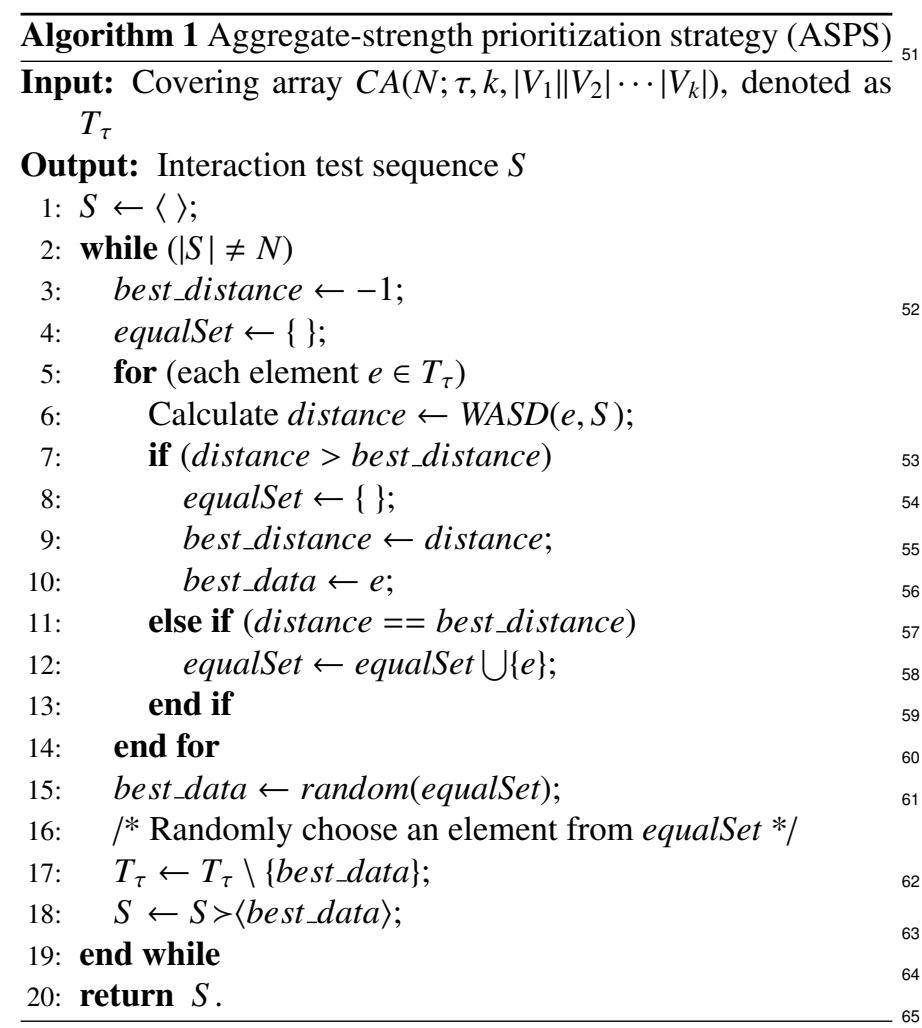

prioritize interaction test suites using the WASD to evaluate combinatorial test cases.

Given a covering array $T=C A\left(N ; \tau, k,\left|V_{1}\right|\left|V_{2}\right| \cdots\left|V_{k}\right|\right)$, the element $e^{\prime}$ is selected from $T$ as the next test element in an interaction test sequence $S$ when using the $W A S D$, such that:

$$
(\forall e)(e \in T)\left(e \neq e^{\prime}\right)\left[\operatorname{WASD}\left(e^{\prime}, S\right) \geq \operatorname{WASD}(e, S)\right] \text {. }
$$

This process is repeated until all candidates have been selected.

The ASPS algorithm is described in Algorithm 1. In some cases, there may be more than one best test element, indicating that they have the same maximal WASD value. In such a situation, a best element is randomly selected.

For ease of description, we use a term $T_{\tau}$ to represent a $C A\left(N ; \tau, k,\left|V_{1}\right|\left|V_{2}\right| \cdots\left|V_{k}\right|\right)$, with the strength $\tau$ used in the WASD and Algorithm 1 often being provided by a CA rather than being assigned in advance.

\subsection{Properties}

Consider a $T_{\tau}$ and a pre-selected interaction test sequence $S \subseteq T_{\tau}$ prioritized by the ASPS algorithm, some properties of ASPS are discussed as follows.

Theorem 1. Once $S$ covers all possible $(\tau-1)$-wise value combinations where $2 \leq \tau \leq k$, the ASPS algorithm has the same mechanism as the "fixed-strength prioritization strategy".

Proof. When $S$ covers all possible $(\tau-1)$-wise value combinations, it can be noted that $\operatorname{CombSet}_{\tau-1}(S)=\operatorname{CombSet}_{\tau-1}\left(T_{\tau}\right)$, which means that

$$
(\forall t c)\left(t c \in\left(T_{\tau} \backslash S\right)\right)\left[U V C D_{\tau-1}(t c, S)=0\right] .
$$

Since

$$
\begin{aligned}
& \operatorname{CombSet}_{\tau-1}(S)=\operatorname{CombSet}_{\tau-1}\left(T_{\tau}\right) \\
\Rightarrow & \operatorname{CombSet}_{\lambda}\left(\operatorname{CombSet}_{\tau-1}(S)\right)=\operatorname{CombSet}_{\lambda}\left(\operatorname{CombSet}_{\tau-1}\left(T_{\tau}\right)\right) \\
\Rightarrow & \operatorname{CombSet}_{\lambda}(S)=\operatorname{CombSet}_{\lambda}\left(T_{\tau}\right),
\end{aligned}
$$

where $1 \leq \lambda \leq \tau-1$. Therefore, we can obtain that

$$
(\forall t c)\left(t c \in\left(T_{\tau} \backslash S\right)\right)\left[U V C D_{\lambda}(t c, S)=0\right] .
$$

where $\lambda=1,2, \cdots, \tau-1 . \quad$ As a consequence, $W A S D(t c, S)=\omega_{\tau} \times \frac{U V C D_{\tau}(t c, S)}{C_{k}^{\tau}}$. Since $\omega_{\tau}$ is a constant parameter, $W A S D(t c, S)$ is only related to $U V C D_{\tau}(t c, S)$. Consequently, the ASPS algorithm only uses $\tau$-wise interaction coverage to select the next test case, which means that it is "fixed-strength prioritization". In summary, once $S$ covers all possible $(\tau-1)$-wise value combinations, the ASPS algorithm becomes the same as "fixed-strength prioritization".

\subsection{Complexity analysis}

In this section, we briefly analyze the complexity of the ASPS algorithm (Algorithm 1). Given a $C A\left(N ; \tau, k,\left|V_{1}\right|\left|V_{2}\right| \cdots\right.$ $\left.\left|V_{k}\right|\right)$, denoted as $T_{\tau}$, we define $\delta=\max _{1 \leq i \leq k}\left\{\left|V_{i}\right|\right\}$. 
We first analyze the time complexity of selecting the $i$-th $(i=1,2, \cdots, N)$ combinatorial test case, which depends on two factors: (1) the number of candidates required for the ${ }^{34}$ calculation of WASD; and (2) the time complexity of ${ }^{35}$ calculating $W A S D$ for each candidate.

For $(1),(N-i)+1$ test cases are required to compute WASD. ${ }^{37}$ For (2), according to $C_{k}^{l} l$-wise parameter combinations where ${ }^{38}$ $1 \leq l \leq \tau$, we divide all $l$-wise value combinations that are ${ }^{39}$ derived from a $T P\left(k,\left|V_{1}\right|\left|V_{2}\right| \cdots\left|V_{k}\right|, \mathscr{D}\right)$ into $C_{k}^{l}$ sets that form ${ }^{40}$

$$
\begin{array}{r}
\Pi_{l}=\left\{\pi_{l} \mid \pi_{l}=\left\{\left(v_{i_{1}}, v_{i_{2}}, \cdots, v_{i_{l}}\right) \mid v_{i_{j}} \in V_{i_{j}}, j=1,2, \cdots, l\right\},\right. \\
\left.1 \leq i_{1}<i_{2}<\cdots<i_{l} \leq k\right\} .
\end{array}
$$

There exists an integer $\eta(1 \leq \eta \leq \tau)$ such that ${ }^{2}$ :

$$
(\forall l)(1 \leq l \leq \tau)(\eta \neq l)\left[\left(C_{k}^{\eta} \times \log \left(\delta^{\eta}\right)\right) \geq\left(C_{k}^{l} \times \log \left(\delta^{l}\right)\right)\right] .
$$

16 As a consequence,

$$
\begin{aligned}
O(A S P S) & <O\left(\frac{N^{2}+N}{2} \times \sum_{l=1}^{\tau}\left(C_{k}^{\eta} \times \log \left(\delta^{\eta}\right)\right)\right) \\
& =O\left(\frac{N^{2}+N}{2} \times \tau \times C_{k}^{\eta} \times \log \left(\delta^{\eta}\right)\right)
\end{aligned}
$$

Therefore, we can conclude that the order of time complexity ${ }_{71}^{70}$ of algorithm ASPS is $O\left(N^{2} \times \tau \times C_{k}^{\eta} \times \log \left(\delta^{\eta}\right)\right)$.

The value of $\tau$ is usually assigned in the range from 2 to $6^{72}$ $[23,24]$, therefore, $1 \leq \tau<\left\lceil\frac{k}{2}\right\rceil$, in general. If $1 \leq \tau<\left\lceil\frac{k}{2}\right\rceil{ }_{74}^{73}$ $\eta=\tau$, then the order of time complexity of algorithm ASPS is ${ }_{75}$ $O\left(\tau \times N^{2} \times C_{k}^{\tau} \times \log \left(\delta^{\tau}\right)\right)$. Since counting the number of value ${ }_{76}$ combinations at different strengths can be implemented in ${ }_{77}$ parallel, the order of time complexity of the ASPS algorithm can be reduced to $O\left(N^{2} \times C_{k}^{\tau} \times \log \left(\delta^{\tau}\right)\right)$. As discussed in [40], the order of time complexity of the ICBP algorithm ( $\mathrm{an}_{80}$ implementation of "fixed-strength prioritization") is $O\left(N^{2} \times C_{k}^{\tau} \times \log \left(\delta^{\tau}\right)\right)$. The order of time complexity of the ${ }_{82}^{81}$ inCTPri algorithm (another "fixed-strength prioritization" ${ }_{83}$ implementation) also equals to $O\left(N^{2} \times C_{k}^{\tau} \times \log \left(\delta^{\tau}\right)\right)$ [39]. ${ }_{84}$ Therefore, the order of time complexity of the ASPS algorithm ${ }_{85}$ is similar to that of both ICBP and inCTPri.

${ }^{2}$ If $1 \leq \tau<\left\lceil\frac{k}{2}\right\rceil, \eta=\tau$; while if $\left\lceil\frac{k}{2}\right\rceil \leq \tau \leq k, \eta=\left\lceil\frac{k}{2}\right\rceil$.

\section{Empirical Study}

In this section, some experimental results, including simulations and experiments involving real programs, are presented to analyze the effectiveness of the prioritization of interaction test suites by multiple interaction coverage (multiple strengths). We evaluate interaction test sequences prioritized by algorithm ASPS (denoted ASPS) by comparing them with those ordered by four other strategies: test-case-generation prioritization (denoted Original), which is an interaction test sequence according to the covering array generation sequence; (2) reverse test-case-generation prioritization (denoted Reverse), which is the reversed order of the Original interaction test sequence; (3) random test sequence, whose ordering is prioritized in a random manner (denoted Random); and (4) two implementations of "fixed-strength prioritization" - the ICBP algorithm (denoted ICBP) $[1,2,4-7,18,30-33,37,40]$; and the inCTPri algorithm (denoted inCTPri) [39].

In Algorithm 1, which uses WASD, it is necessary to assign a weight for each interaction coverage (Eq. (7)). The ideal weight assignment is in accordance with actual fault distribution in terms of the FTFI number. However, the actual fault distribution is unknown before testing. In this paper, we focus on three distribution styles: (1) equal weighting distribution - each interaction coverage has the same weight, that is, $\omega_{1}=\omega_{2}=\cdots=\omega_{\tau}=\frac{1}{\tau}$; (2) random weighting distribution - the weight of each interaction coverage is randomly distributed; and (3) empirical FTFI percentage weighting distribution based on previous investigations [23, 24]: for example, in [24], several software projects were studied and the interaction faults reported to have $29 \%$ to $82 \%$ faults as 1 -wise faults, $6 \%$ to $47 \%$ of faults as 2 -wise faults, $2 \%$ to $19 \%$ as 3 -wise faults, $1 \%$ to $7 \%$ of faults as 4 -wise faults, and even fewer faults beyond 4-wise interactions. Consequently, we arranged the weights as follows: $\omega_{1}=\omega, \omega_{i+1}=\frac{1}{2} \omega_{i}$, where $i=1,2, \cdots, \tau-1$. For example, if $\tau=2$, we set $\omega_{1}=0.67$ and $\omega_{2}=0.33$; if $\tau=3, \omega_{1}=0.57$, $\omega_{2}=0.29$, and $\omega_{3}=0.14$. For clarity of description, we use $\mathrm{ASPS}_{e}, \mathrm{ASPS}_{r}$, and $\mathrm{ASPS}_{m}$ to represent the ASPS algorithm with equal weighting distribution, random weighting distribution, and empirical FTFI percentage weighting distribution, respectively.

The original covering arrays were generated using two popular tools: (1) Advanced Combinatorial Testing System (ACTS) [25, 38]; and (2) Pairwise Independent Combinatorial Testing (PICT) [10]. Both ACTS and PICT are supported by greedy algorithms, however, they are implemented by different strategies: ACTS is implemented by the In-Parameter-Order (IPO) method [38]; while PICT is implemented by the one-test-at-a-time approach [3]. We focused on covering arrays with strength $\tau=2,3,4,5$. We designed simulations and experiments to answer the following research questions:

RQ1: Do prioritized interaction test sequences generated by ASPS methods $\left(\mathrm{ASPS}_{e}, \mathrm{ASPS}_{r}\right.$, and $\mathrm{ASPS}_{m}$ ) have better performance (e.g. fault detection) when compared with non-prioritized interaction test suites (Original)? The answer 
Table 3: Sizes of covering arrays for four test profiles.

\begin{tabular}{|c|c|c|c|c|c|c|c|c|}
\hline \multirow{2}{*}{ Test Profile } & \multicolumn{4}{|c|}{ ACTS } & \multicolumn{4}{|c|}{ PICT } \\
\hline & $\tau=2$ & $\tau=3$ & $\tau=4$ & $\tau=5$ & $\tau=2$ & $\tau=3$ & $\tau=4$ & $\tau=5$ \\
\hline$T P 1\left(6,5^{6}, \emptyset\right)$ & 25 & 199 & 1058 & 4149 & 37 & 215 & 1072 & 4295 \\
\hline$T P 2\left(10,2^{3} 3^{3} 4^{3} 5^{1}, \emptyset\right)$ & 23 & 103 & 426 & 1559 & 23 & 109 & 411 & 1363 \\
\hline$T P 3\left(7,2^{4} 3^{1} 6^{1} 16^{1}, \emptyset\right)$ & 96 & 289 & 578 & 1728 & 96 & 293 & 744 & 1658 \\
\hline$T P 4\left(8,2^{6} 9^{1} 10^{1}, \emptyset\right)$ & 90 & 180 & 632 & 1080 & 90 & 192 & 592 & 1237 \\
\hline
\end{tabular}

to this question will help us decide whether it would be ${ }_{41}$ necessary to prioritize interaction test suites using ASPS $_{42}$ methods.

RQ2: Are ASPS methods better than intuitive ${ }_{44}$ prioritization strategies such as the reverse prioritization ${ }_{45}$ (Reverse) and the random prioritization (Random)? The ${ }_{46}$ answer to this question will tell us whether or not it would be ${ }_{47}$ helpful to use ASPS methods rather than reverse or random ${ }_{48}$ ordering for prioritizing interaction test suites.

RQ3: Do ASPS methods perform better than "fixed-strength prioritization" (ICBP and inCTPri)? The answer to this question will help us decide whether or not ASPS can be a promising technique for interaction test suite prioritization, especially if it could perform as effectively as ${ }^{50}$ the current best prioritization techniques ("fixed-strength" prioritization").

RQ4: Which weighting distribution is more suitable for ${ }^{53}$ the ASPS method: equal weighting distribution, random ${ }^{54}$ weighting distribution, or empirical FTFI percentage weighting distribution? The answer to this question will help ${ }^{56}$ us decide which weighting distribution to use for the ASPS method.

\subsection{Simulation}

We ran a simulation to measure how quickly an interaction ${ }^{58}$ test sequence could cover value combinations of different ${ }^{59}$ strengths. The simulation details are presented in the following.

\subsubsection{Setup}

We designed four test profiles as four system models with ${ }^{64}$ details as shown in Table 3 . The first two test profiles were ${ }^{65}$ $T P 1\left(6,5^{6}, \emptyset\right)$ and $T P 2\left(10,2^{3} 3^{3} 4^{3} 5^{1}, \emptyset\right)$, both of which have ${ }^{66}$ been used in previous studies [40]. The third and fourth test ${ }_{68}^{67}$ profiles $\left(T P 3\left(7,2^{4} 3^{1} 6^{1} 16^{1}, \emptyset\right)\right.$ and $\left.T P 4\left(8,2^{6} 9^{1} 10^{1}, \emptyset\right)\right)$ have previously been created $[32,33]$ to model real-world subjects - a module from a lexical analyzer system (flex), and a real ${ }^{70}$ configuration model for GNUzip (gzip) $)^{3}$.

The sizes of the covering arrays generated by ACTS and PICT are given in Table 3, Since randomization is used in some test case prioritization techniques, we ran each test profile 100 times and report the average of the results.

\footnotetext{
${ }^{3}$ These two models are unconstrained and incomplete.
}

\subsubsection{Metric}

The average percentage of combinatorial coverage (APCC) metric ${ }^{4}$ [40] is used to evaluate the rate of value combinations covered by an interaction test sequence. The APCC values range from $0 \%$ to $100 \%$, with higher values meaning better rates of covering value combinations. Let an interaction test sequence $S=\left\langle s_{1}, s_{2}, \cdots, s_{N}\right\rangle$ be obtained by prioritizing a $C A\left(N ; \tau, k,\left|V_{1}\right|\left|V_{2}\right| \cdots\left|V_{k}\right|\right)$, that is, $T_{\tau}$, the formula to calculate APCC at strength $\lambda(1 \leq \lambda \leq \tau)$ is:

$$
\operatorname{APCC}_{\lambda}(S)=\frac{\sum_{i=1}^{N-1}\left|\bigcup_{j=1}^{i} \operatorname{CombSet}_{\lambda}\left(s_{j}\right)\right|}{N \times\left|\operatorname{CombSet}_{\lambda}\left(T_{\tau}\right)\right|} \times 100 \% .
$$

Additionally, since we consider $\lambda=1,2, \cdots, \tau$ for an interaction test sequence $S$ of a $C A\left(N ; \tau, k,\left|V_{1}\right|\left|V_{2}\right| \cdots\left|V_{k}\right|\right)$, we could obtain $\tau$ APCC values (that is, $A P C C_{1}(S), A P C C_{2}(S)$, $\left.\ldots, A P C C_{\tau}(S)\right)$. Therefore, in this simulation we also considered the average of the APCC values, which is defined as:

$$
\operatorname{Avg.}(S)=\frac{1}{\tau} \sum_{\lambda=1}^{\tau} A P C C_{\lambda}(S) \text {. }
$$

\subsubsection{Results and Discussion}

For covering arrays of strength $\tau(2 \leq \tau \leq 5)$ on individual test profiles, we have the following observations based on the data in Tables 4 and 5, which are separated according to the four test profiles.

1) According to the APCC metric, are prioritized interaction test suites by ASPS methods better than non-prioritized interaction test suites? In this part, we analyze the data to answer whether ASPS methods $\left(\operatorname{ASPS}_{e}, \mathrm{ASPS}_{r}\right.$, and $\mathrm{ASPS}_{m}$ ) are more effective than Original.

Since different weighting distributions in ASPS provide different APCC values, we compare the APCC values of each ASPS method with Original. In 98.21\% (110/112), $86.61 \%(97 / 112)$, and $98.21 \%(110 / 112)$ of cases, interaction test sequences prioritized by $\mathrm{ASPS}_{e}, \mathrm{ASPS}_{r}$, and $\mathrm{ASPS}_{m}$,

\footnotetext{
${ }^{4}$ In [30], Petke et al. proposed a similar metric, namely the average percentage of covering-array coverage metric (APCC). Both Wang's APCC [40] and Petke's APCC [30] aim at measuring how quickly an interaction test sequence achieves interaction coverage at a given strength. In fact, their APCCs are equivalent: given two interaction test sequences, $S_{1}$ and $S_{2}$, if one determines that the test sequence $S_{1}$ is better than $S_{2}$, then the other metric will also have the same determination. The only difference between them is that they use different plot curves to describe the rate of covered value combinations. More specifically, Wang's APCC [40] uses the ladder chart; while Petke's APCC [30] uses the line chart.
} 


\begin{tabular}{|c|c|c|c|c|c|}
\hline & $\frac{80}{\pi}$ & 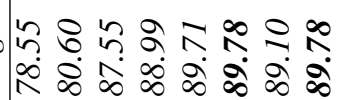 & 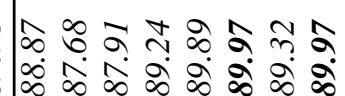 & 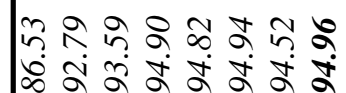 & 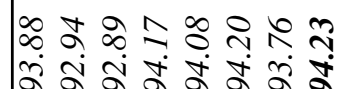 \\
\hline & $\stackrel{n}{\sim}$ & 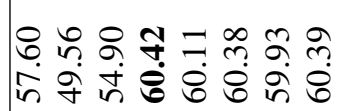 & 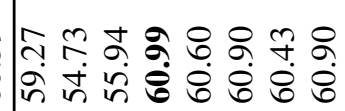 & 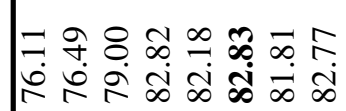 & 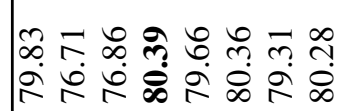 \\
\hline & II & 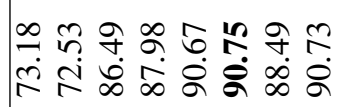 & 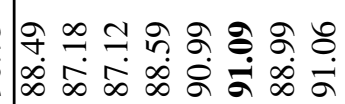 & 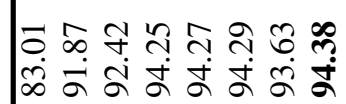 & 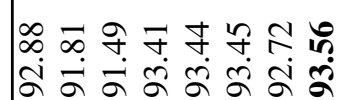 \\
\hline & $\stackrel{\pi}{\sim}$ & 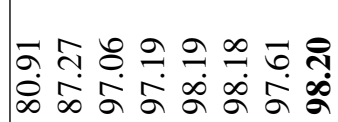 & 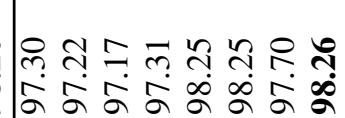 & 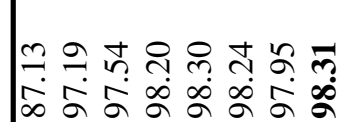 & 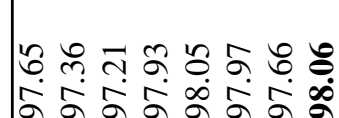 \\
\hline & $\stackrel{\mathbb{1}}{2}$ & 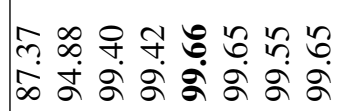 & 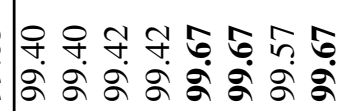 & 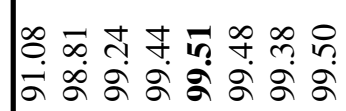 & 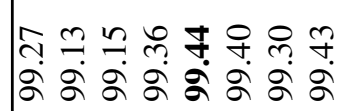 \\
\hline & II & 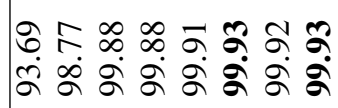 & 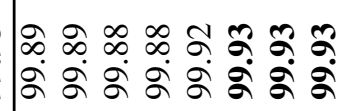 & 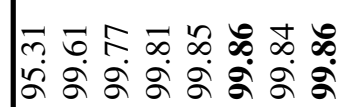 & 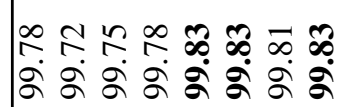 \\
\hline & $\dot{\infty}$ & 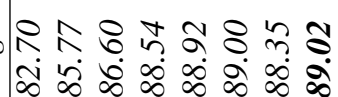 & 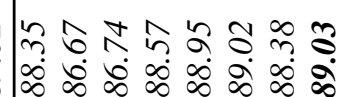 & 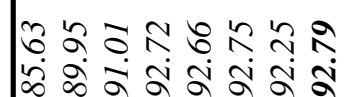 & 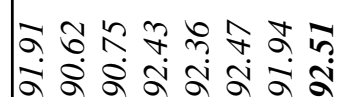 \\
\hline & II & जि: & 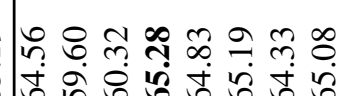 & 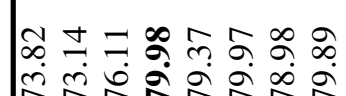 & 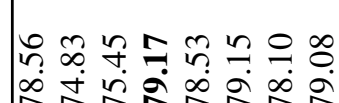 \\
\hline & & & & & \\
\hline & III & 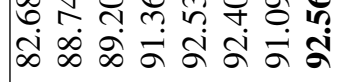 & 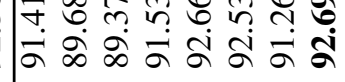 & बूँ & ๙ু \\
\hline & $\stackrel{N}{\sim}$ & 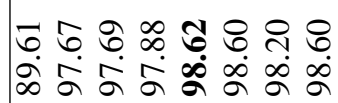 & 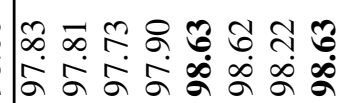 & 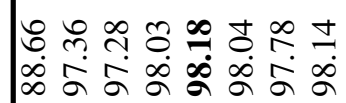 & 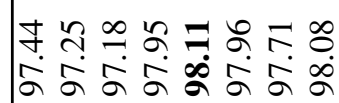 \\
\hline & II & 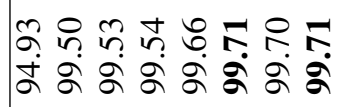 & 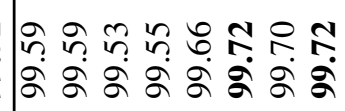 & 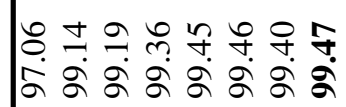 & 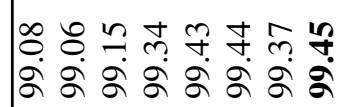 \\
\hline & $\dot{\infty}$ & 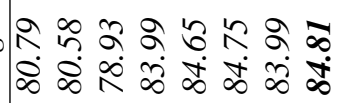 & 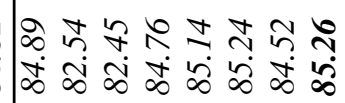 & 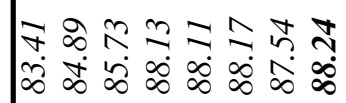 & 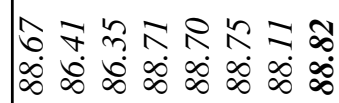 \\
\hline & II & 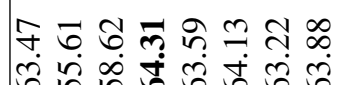 & 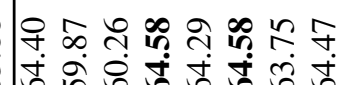 & 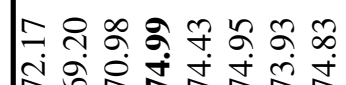 & 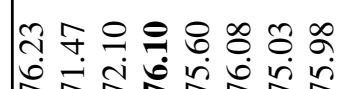 \\
\hline & $\stackrel{\pi}{\sim}$ & 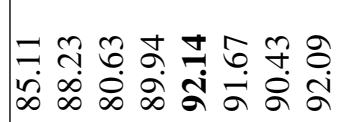 & 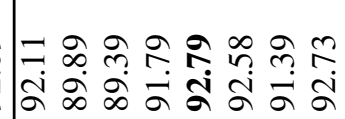 & 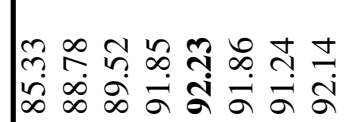 & 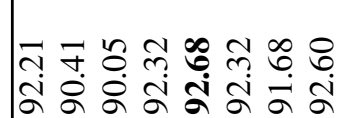 \\
\hline & II & 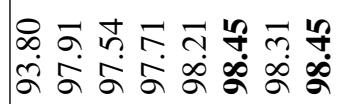 & 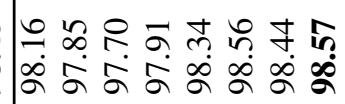 & 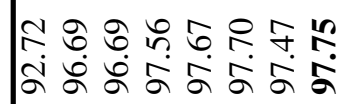 & 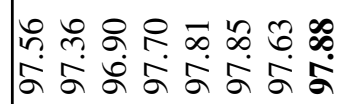 \\
\hline & $\dot{\infty}$ & 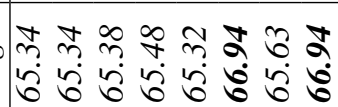 & 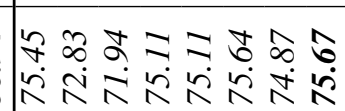 & 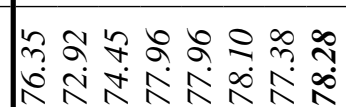 & 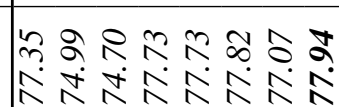 \\
\hline & & 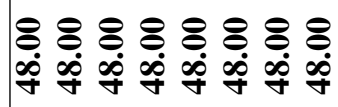 & 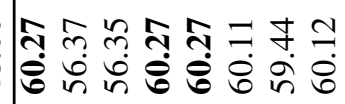 & 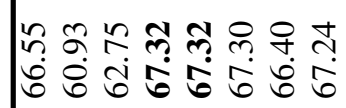 & 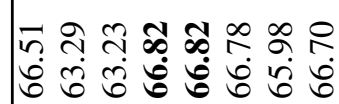 \\
\hline & II & 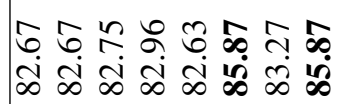 & 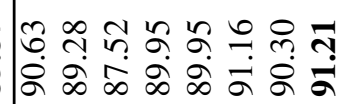 & 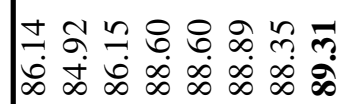 & 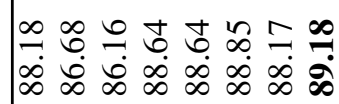 \\
\hline & $\begin{array}{l}\text { D } \\
\text { 帝 } \\
\sum\end{array}$ & 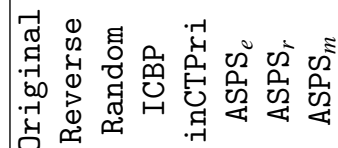 & 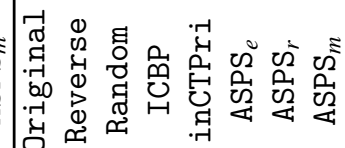 & 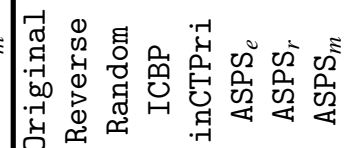 & 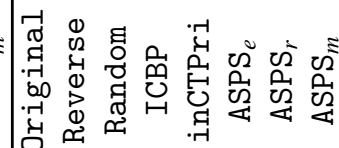 \\
\hline & & $S L D V-I d L$ & $L \partial I d-I d L$ & $S L \partial V-Z_{d} L$ & $L \supset I d-\tau_{d} L$ \\
\hline
\end{tabular}




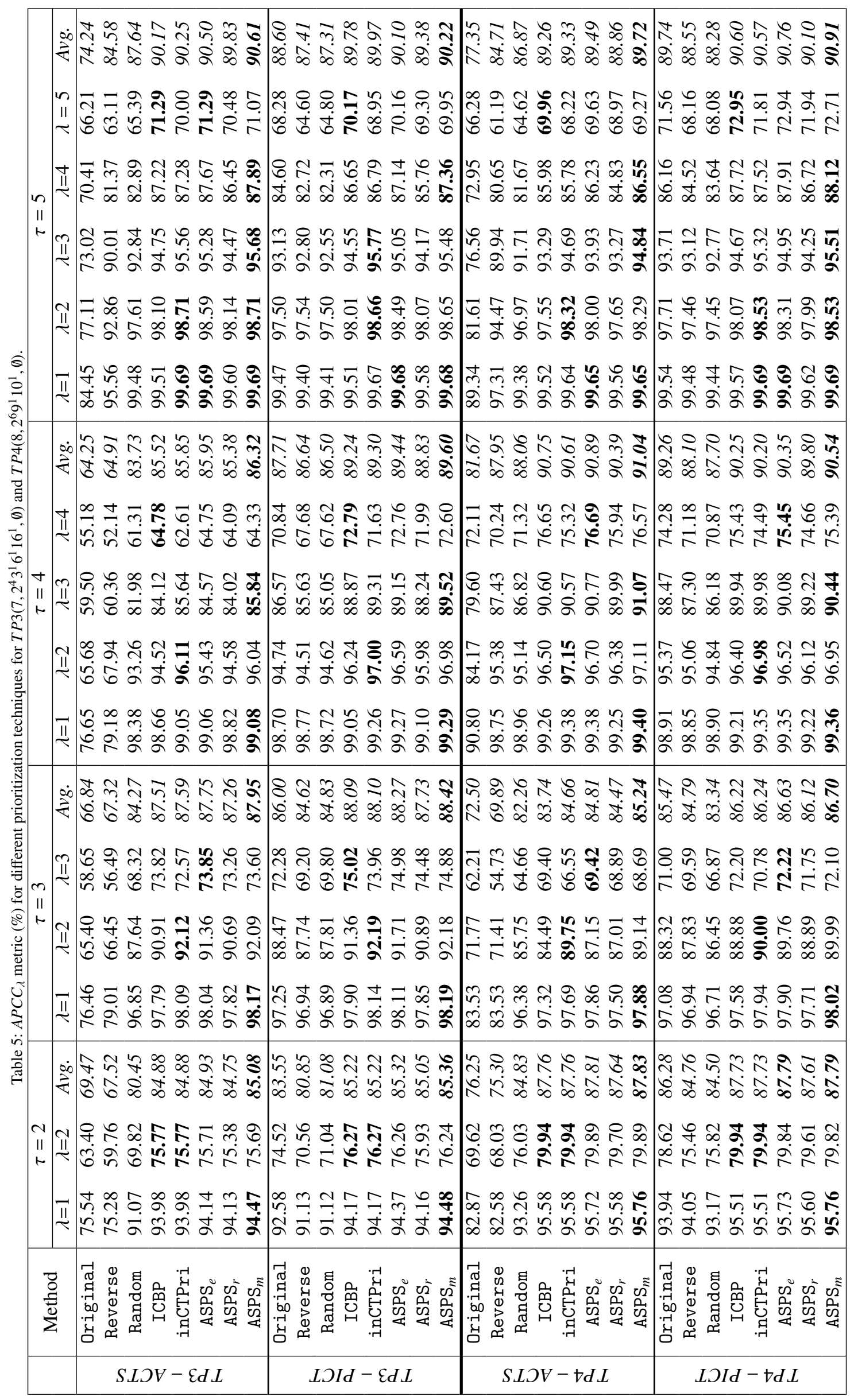


respectively, have greater APCC values than Original test sequences. Additionally, the average APCC values of $\mathrm{ASPS}_{e}$, $\mathrm{ASPS}_{r}$, and $\mathrm{ASPS}_{m}$ are higher than those of Original, in $100.00 \%(32 / 32), 84.38 \%(27 / 32)$, and $100.00 \%(32 / 32)$ of cases, respectively.

As shown in Tables 4 and 5, it can be noted that different non-prioritized interaction test suites generated by different tools have different performances.

For example, consider $T P 3\left(7,2^{4} 3^{1} 6^{1} 16^{1}, \emptyset\right)$ at strength $\tau=2$ : when non-prioritized covering arrays are constructed using ACTS, the difference between $\mathrm{ASPS}_{e}$ and Original is $18.60 \%$ for $\lambda=1$, and $32.31 \%$ for $\lambda=2$. However, when using PICT, the difference is $1.79 \%$ for $\lambda=1$, and $1.74 \%$ for $\lambda=2$. The main reason for this is related to the different 58 mechanisms used in the ACTS and PICT tools [10, 25, 38]. 59 Specifically, without loss of generality, consider a test profile 60 $T P\left(k,\left|V_{1}\right|\left|V_{2}\right| \cdots\left|V_{k}\right|, \emptyset\right)$ with $\left|V_{1}\right| \geq\left|V_{2}\right| \geq \cdots \geq\left|V_{k}\right|$. When ${ }_{61}$ generating a $\tau$-wise $(1 \leq \tau \leq k)$ covering array, the ACTS ${ }_{62}$ algorithm first uses horizontal growth $[25,38]$ to construct a 63 $\tau$-wise test set for the first $\tau$ parameters, which implies that it ${ }_{64}$ needs at least $\left(1+\left(\left|V_{1}\right|-1\right) \times \prod_{i=2}^{\tau}\left|V_{i}\right|\right)$ test cases to cover all ${ }_{65}$ possible 1-wise value combinations. However, the PICT 66 algorithm chooses each next test case such that it covers the ${ }_{67}$ largest number of $\tau$-wise value combinations that have not yet ${ }_{68}$ been covered - a mechanism similar to that of ICBP.

In conclusion, the simulation indicates that the ASPS 70 techniques do outperform Original in terms of the rate of 71 covering value combinations, regardless of which construction 72 tools are used (ACTS or PICT).

2) Do ASPS methods have better APCC values than 74 reverse or random ordering? In this part, we attempt to 75 determine whether or not ASPS methods are more effective 76 than two widely-used prioritization methods, Reverse and 77 Random.

In all cases, each ASPS method (regardless of $\mathrm{ASPS}_{e}, 79$ $\operatorname{ASPS}_{r}$, and $\mathrm{ASPS}_{m}$ ) has higher APCC values than Reverse, 80 and hence achieves higher average APCC values. Additionally, 81 the performance of Reverse is correlated with the ${ }_{82}$ non-prioritized interaction test suite (that is, the ACTS and ${ }_{83}$ PICT tools).

Compared with Random, the ASPS methods have higher ${ }_{85}$ APCC values in all cases, irrespective of the strength 86 $\lambda=1,2, \cdots, \tau$ and interaction test suite construction tool 87 (ACTS or PICT). As a result, the ASPS methods have better 88 performance according to the average APCC values at ${ }_{89}$ different strengths.

In conclusion, in all cases our ASPS methods (regardless 91 of the weighting distributions) do perform better than both the 92 Reverse and Random prioritization strategies, according to the 93 APCC values.

3) Are ASPS methods better than "fixed-strength prioritization"? In this part, we would like to determine 96 whether or not ASPS methods perform better than two 97 implementations of "fixed-strength prioritization", ICBP and inCTPri.

Compared with ICBP, according to APCC values, $\mathrm{ASPS}_{e}$, ASPS $_{r}$ and $\mathrm{ASPS}_{m}$ perform better in $79.46 \%$ (89/112),

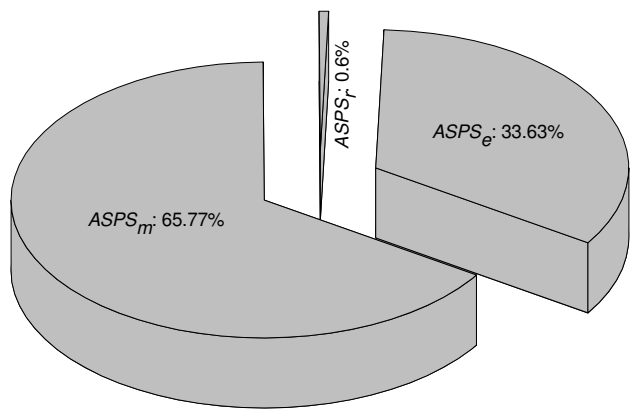

Figure 1: Comparison of $\mathrm{ASPS}_{e}, \mathrm{ASPS}_{r}$, and $\mathrm{ASPS}_{m}$ according to APCC.

$36.61 \%(41 / 112)$, and $72.32 \%(81 / 112)$ of cases, respectively. Furthermore, according to the average of APCC values (Avg.), $\mathrm{ASPS}_{e}, \mathrm{ASPS}_{r}$, and $\mathrm{ASPS}_{m}$ have better performances than ICBP in $100.00 \%(32 / 32), 18.75 \%(6 / 32)$, and $100.00 \%(32 / 32)$ of cases.

Similarly, compared with inCTPri, $\mathrm{ASPS}_{e}, \mathrm{ASPS}_{r}$, and ASPS $_{m}$ have higher APCC values in 58.93\% (66/112) $21.43 \%(24 / 112)$, and $75.00 \%$ (84/112) of cases, respectively Moreover, according to the average of APCC values, $\mathrm{ASPS}_{e}$, $\mathrm{ASPS}_{r}$, and $\mathrm{ASPS}_{m}$ outperform inCTPri in $100.00 \%(32 / 32)$, $3.13 \%(1 / 32)$, and $100.00 \%(32 / 32)$ of cases.

In conclusion, the simulation results indicate that apart from $\mathrm{ASPS}_{r}$, in $(58.93 \% \sim 79.46 \%)$ of cases our ASPS methods $\left(\mathrm{ASPS}_{e}\right.$ and $\left.\mathrm{ASPS}_{m}\right)$ do perform better than ICBP and inCTPri, and also have higher averages of APCC values in all cases. Consequently, we conclude that our ASPS methods (except for $\mathrm{ASPS}_{r}$ ) do have better performance than "fixed-strength prioritization".

4) Among the three weighting distributions, which weighting distribution is used for the ASPS method? In this part, we are interested in which weighting distribution is more suitable for the ASPS method. As discussed before, there are three distributions used for the ASPS methods: equal, random, and empirical FTFI percentage weighting distributions.

As discussed in the last part, among the three weight distributions for ASPS, $\mathrm{ASPS}_{r}$ has the lowest $A P C C_{\lambda}$ performance, irrespective of which $\lambda$ value is used $(1 \leq \lambda \leq \tau)$. Additionally, when $\lambda$ is high, $\operatorname{ASPS}_{e}$ performs better than $\mathrm{ASPS}_{m}$, otherwise it performs worse. According to the average APCC values, however, $\operatorname{ASPS}_{m}$ performs best, followed by $\mathrm{ASPS}_{e}$; while $\mathrm{ASPS}_{r}$ has the worst performance. Figure 1 shows the comparison of $\mathrm{ASPS}_{e}, \mathrm{ASPS}_{r}$, and $\mathrm{ASPS}_{m}$ according to the APCC metric. From this figure, it can be observed that in $65.77 \%(73.67 / 112)$ of cases $\mathrm{ASPS}_{m}$ has the highest APCC values; in $33.63 \%(37.67 / 112)$ of cases, ASPS $_{e}$ does; and onl in $0.60 \%(0.67 / 112)$ of cases is the highest values for $\operatorname{ASPS}_{r}{ }^{5}$. In other words, among three weighting distributions, empirical FTFI percentage weighting distribution would be the best choice, followed by the equal weighting distribution, according to the APCC metric.

${ }^{5}$ If there exist two or three ASPS strategies that hav 5 same APCC value, each strategy is assigned by $1 / 2$ or $1 / 3$. 
Table 6: Subject programs.

\begin{tabular}{cccccc}
\hline Subject Program & Test Profile & \#uLOC & \#Seeded Faults & \#Detectable Faults & \#Used Faults \\
\hline count & $T P\left(6,2^{1} 3^{5}, \emptyset\right)$ & 42 & 15 & 12 & 12 \\
nametbl & $T P\left(5,2^{1} 3^{2} 5^{2}, \emptyset\right)$ & 329 & 51 & 44 & 43 \\
\hline flex & $T P\left(9,2^{6} 3^{2} 5^{1}, \mathscr{D}\right)$, & $9,581 \sim 11,470$ & 81 & 50 & 34 \\
grep & $T P\left(9,2^{1} 3^{3} 4^{2} 5^{1} 6^{1} 8^{1}, \mathscr{D}\right)$ & $9,493 \sim 10,173$ & 57 & 12 & 10 \\
\hline
\end{tabular}

In conclusion, the empirical FTFI percentage weighting ${ }_{36}$ distribution appears to be more suitable than the other ${ }_{37}$ weighting distributions for the ASPS method ( $\left.\operatorname{ASPS}_{m}\right)$.

5) Conclusion: Based on the above discussions, we find ${ }_{39}$ that given a covering array of strength $\tau$, the ASPS strategies 40 behave better than the Original, Reverse, and Random ${ }_{41}$ strategies (in $86.61 \% \sim 100.00 \%$ of cases), and apart from ${ }_{42}$ $\mathrm{ASPS}_{r}$ perform better than "fixed-strength prioritization" 43 including ICBP and inCTPri implementations (in 44 $58.93 \% \sim 79.46 \%$ of cases). Additionally, among the three 45 weighting distributions, the empirical FTFI percentage is most 46 suitable for use with the ASPS method, followed by the equal 47 weighting distribution.

\subsection{Experiments}

An experimental study was also conducted to evaluate the ${ }_{51}$ ASPS techniques, the goal of which was to compare the fault ${ }_{52}$ detection rates of the $\mathrm{ASPS}_{e}, \mathrm{ASPS}_{r}$, and $\mathrm{ASPS}_{m}$ techniques ${ }_{53}$ against those of other interaction test suite prioritization ${ }_{54}$ techniques, such as Original, Reverse, Random, ICBP, and ${ }_{55}$ inCTPri. In actual testing conditions, testing resources may ${ }_{56}$ be limited, and hence only part of an interaction test suite (or ${ }_{57}$ an interaction test sequence) may be executed. As $a_{58}$ consequence, in this study we focused on different budgets by ${ }_{59}$ following the practice adopted in previous prioritization ${ }_{60}$ studies [30] of considering different percentages $(p)$ of each ${ }_{61}$ interaction test sequence, e.g. $p=5 \%, 10 \%, 25 \%, 50 \%, 75 \%$, and $100 \%$ of each interaction test sequence being executed.

\subsubsection{Setup}

For the study, we used two small-sized faulty $\mathrm{C}$ programs (count and nametbl) ${ }^{6}$, which had previously been used in ${ }_{66}^{65}$ research comparing defect revealing mechanisms [27], ${ }_{67}^{66}$ evaluating different combination strategies for test case selection [17], and fault diagnosis [16, 45]. Since these two programs are small, we also used another two medium-sized UNIX utility programs ${ }^{7}$, flex and grep, with real and seeded

${ }^{6}$ From http://www . maultech.com/chrislott/work/exp/

${ }^{7}$ From http://sir.unl.edu faults from the Software-artifact Infrastructure Repository (SIR) [11]. These two programs had also been widely used in prioritization research $[22,30,32]$. To determine the correctness of an executing test case, we created a fault-free version of each program (i.e. an oracle) by analyzing the corresponding fault description. These subject programs are described in Table 6, in which the Test Profile is the test profile of each subject program ${ }^{8}$; the $\# u L O C^{9}$ gives the number of lines of executable code in each program; the \#Seeded Faults ${ }^{10}$ is the number of faults seeded in each program; the \#Detectable Faults is the number of faults that could be detected from the accompanying test profiles, which are not guaranteed to be able to detect all faults; and the \#Used Faults is the number of faults used in the experiment by removing some faults that could be triggered by nearly every combinatorial test case in the test suite, that is, faults could be removed such that they are identified by more than $(78.00 \% \sim 100.00 \%)$ test cases in the test suite.

Similar to the simulation (Section 5.1), we also used ACTS and PICT to generate original interaction test sequences for each subject program. Additionally, we focused on covering arrays with strength $\tau=2,3,4,5$. Table 7 gives the sizes of the original interaction test sequences obtained by ACTS and PICT. Because of the randomization in some of the prioritization techniques, we ran the experiment 100 times for each subject program and report the average.

\subsubsection{Metric}

The APFD metric [13] is a popular measure for evaluating fault detection rates of interaction test sequence. In this study, only part of the interaction test sequences could be run, and some faults might not have been triggered by a particular interaction test sequence. As discussed before, however,

\footnotetext{
${ }^{8}$ The test profiles of two medium-sized programs, flex and grep, are from Petke et al. [30].

${ }^{9} \mathrm{We}$ used the line count tool named cloc, downloaded from http://cloc.sourceforge.net, to count the number of code lines.

${ }^{10}$ Similar to [30], in this study we only used the faults provided with each of subject programs, in order to avoid experiment bias and ensure repeatability.
}

Table 7: Sizes of original interaction test sequences for each subject program.

\begin{tabular}{|c|c|c|c|c|c|c|c|c|}
\hline \multirow{2}{*}{ Subject Program } & \multicolumn{4}{|c|}{ ACTS } & \multicolumn{4}{|c|}{ PICT } \\
\hline & $\tau=2$ & $\tau=3$ & $\tau=4$ & $\tau=5$ & $\tau=2$ & $\tau=3$ & $\tau=4$ & $\tau=5$ \\
\hline count & 15 & 41 & 108 & 243 & 14 & 43 & 116 & 259 \\
\hline nametbl & 25 & 82 & 225 & 450 & 25 & 78 & 226 & 450 \\
\hline flex & 27 & 66 & 130 & 238 & 26 & 65 & 129 & 219 \\
\hline grep & 46 & 153 & 298 & 436 & 47 & 150 & 296 & 436 \\
\hline
\end{tabular}


APFD has two requirements which may cause APFD to fail. 19 Consequently, it was not possible to use APFD to investigate 20 the fault detection rates of the different prioritization strategies, 21 and so we used an enhanced version of APFD, NAPFD [32], 22 as an alternative evaluation metric.

\subsubsection{Results and Discussions}

The experimental results from executing all prioritization 26 techniques to test count, nametbl, flex, and grep are 27 summarized in Tables $8 \sim 11$, based on which we can have the 28 following observations. It should be noted that the data in bold 29 in the tables is the largest in each sub-column.

1) Does the ASPS method have faster fault detection rates 31 than the Original method? In this part, we analyze the 32 experimental data to answer the research question of whether 33 or not the ASPS method is better than Original according to 34 fault detection rates.

As shown in Tables $8 \sim 11$, in 96.84\% (184/190), 36 $97.37 \%(185 / 190)$, and $96.84 \%(184 / 190)$ of cases, $\mathrm{ASPS}_{e},{ }^{37}$
$\mathrm{ASPS}_{r}$, and $\mathrm{ASPS}_{m}$, respectively, obtain interaction test sequences with higher fault detection rates than Original. The fault detection improvement of ASPS over Original for ACTS is larger than that for PICT: as was the case in the simulation, the main reason for this is the different methods used to construct ACTS and PICT interaction test suites.

Additionally, as the proportion of the interaction test sequence executed $(p)$ increases, the NAPFD improvement of ASPS over Original generally becomes smaller. For example, consider subject program nametbl for ACTS with $\tau=5$, when $p=5 \%, 10 \%, 25 \%, 50 \%, 75 \%$, and $100 \%$, the corresponding NAPFD improvements of $\mathrm{ASPS}_{e}$ over Original are $58.92 \%, 42.87 \%, 27.78 \%, 14.54 \%, 9.71 \%$, and $7.27 \%$, respectively.

In conclusion, in $(97.37 \% \sim 96.84 \%)$ of cases, the ASPS method has higher rates of fault detection compared with Original. Furthermore, the ASPS method favors the cases where smaller percentages of interaction test sequence are executed, compared with Original.

Table 8: The NAPFD metric (\%) for different prioritization techniques for subject program count when executing the percentage of interaction test sequence.

\begin{tabular}{|c|c|c|c|c|c|c|c|c|c|c|c|c|c|}
\hline \multirow{2}{*}{ Method } & \multirow{2}{*}{ Strength } & \multicolumn{6}{|c|}{$p$ of ACTS Interaction Test Sequence Executed } & \multicolumn{6}{|c|}{$p$ of PICT Interaction Test Sequence Executed } \\
\hline & & $5 \%$ & $10 \%$ & $25 \%$ & $50 \%$ & $75 \%$ & $100 \%$ & $5 \%$ & $10 \%$ & $25 \%$ & $50 \%$ & $75 \%$ & $100 \%$ \\
\hline Original & \multirow{8}{*}{$\tau=2$} & - & 0 & 0 & 25.00 & 48.11 & 59.72 & - & 0 & 33.33 & 69.05 & 78.33 & 84.52 \\
\hline Reverse & & - & 0 & 30.56 & 62.50 & 73.11 & 78.06 & - & 25.00 & 43.06 & 51.79 & 60.00 & 71.43 \\
\hline Random & & - & 15.38 & 37.75 & 60.08 & 70.76 & 76.31 & - & 17.71 & 44.18 & 68.19 & 76.71 & 83.21 \\
\hline ICBP & & - & 16.29 & 40.56 & 61.98 & 72.05 & 77.27 & - & 17.96 & 46.67 & 72.69 & 80.82 & 86.30 \\
\hline inCTPri & & - & 16.29 & 40.56 & 61.98 & 72.05 & 77.27 & - & 17.96 & 46.67 & 72.69 & 80.82 & 86.30 \\
\hline $\mathrm{ASPS}_{e}$ & & - & 15.21 & 38.81 & 62.32 & 72.09 & 77.29 & - & 19.04 & 50.68 & 75.45 & 82.82 & 87.73 \\
\hline $\mathrm{ASPS}_{r}$ & & - & 17.83 & 42.69 & 63.86 & 73.18 & 78.10 & - & 17.08 & 47.72 & 72.81 & 80.62 & 86.15 \\
\hline $\operatorname{ASPS}_{m}$ & & - & 13.58 & 39.10 & 62.90 & 72.32 & 77.45 & - & 17.13 & 47.24 & 73.99 & 81.79 & 86.99 \\
\hline Original & \multirow{8}{*}{$\tau=3$} & 0 & 0 & 1.25 & 31.04 & 53.33 & 65.85 & 12.50 & 34.38 & 65.00 & 83.33 & 89.06 & 91.86 \\
\hline Reverse & & 39.58 & 48.96 & 58.75 & 74.58 & 83.06 & 87.60 & 0 & 21.88 & 55.83 & 78.97 & 86.20 & 89.73 \\
\hline Random & & 0.40 & 47.89 & 71.23 & 84.03 & 89.08 & 91.98 & 29.96 & 46.75 & 71.65 & 85.79 & 90.67 & 93.06 \\
\hline ICBP & & 9.06 & 48.25 & 73.25 & 86.03 & 90.64 & 93.15 & 27.25 & 48.43 & 75.91 & 88.49 & 92.45 & 94.38 \\
\hline inCTPri & & 31.71 & 51.96 & 75.85 & 87.05 & 91.34 & 93.66 & 32.98 & 54.44 & 78.45 & 89.68 & 93.23 & 94.96 \\
\hline $\mathrm{ASPS}_{e}$ & & 32.63 & 54.40 & 75.98 & 86.83 & 91.16 & 93.53 & 30.92 & 54.42 & 78.92 & 89.90 & 93.37 & 95.07 \\
\hline $\mathrm{ASPS}_{r}$ & & 33.21 & 53.60 & 75.45 & 86.68 & 91.09 & 93.48 & 34.42 & 53.15 & 77.06 & 88.74 & 92.61 & 94.50 \\
\hline $\mathrm{ASPS}_{m}$ & & 35.00 & 55.78 & 76.39 & 86.94 & 91.23 & 93.58 & 30.90 & 54.75 & 78.74 & 89.85 & 93.34 & 95.04 \\
\hline Original & \multirow{8}{*}{$\tau=4$} & 0 & 0 & 0 & 28.86 & 51.03 & 63.27 & & 80.30 & 92.53 & 96.26 & 97.51 & 98.13 \\
\hline Reverse & & 65.00 & 82.50 & 93.52 & 96.76 & 97.84 & 98.38 & 74.17 & 87.12 & 95.11 & 97.56 & 98.37 & 98.78 \\
\hline Random & & & & & & 96.32 & & & & 98 & 94.49 & .33 & 97.25 \\
\hline ICBP & & 56.04 & 74.30 & 90.15 & 95.08 & 96.72 & 97.54 & 52.76 & 74.07 & 89.96 & 94.98 & 96.65 & 97.49 \\
\hline inCTPri & & & 77.81 & 91.52 & 95.76 & 97.17 & 97.88 & & 79.42 & 92.04 & 96.02 & 97.35 & 98.01 \\
\hline $\mathrm{ASPS}_{e}$ & & 61.80 & 78.25 & 91.78 & 95.89 & 97.26 & 97.95 & 60.28 & 79.11 & 91.94 & 95.97 & 97.31 & 97.99 \\
\hline $\mathrm{ASPS}_{r}$ & & 60.67 & 77.70 & 91.40 & 95.69 & 97.13 & 97.85 & 60.85 & 78.61 & 91.41 & 95.71 & 97.14 & 97.85 \\
\hline $\mathrm{ASPS}_{m}$ & & 61.63 & 78.34 & 91.64 & 95.82 & 97.21 & 97.91 & 60.39 & 79.32 & 91.94 & 95.97 & 97.31 & 97.98 \\
\hline Original & \multirow{8}{*}{$\tau=5$} & 0 & 0 & 0 & 16.53 & 35.92 & 51.75 & 70.14 & 85.67 & 94.40 & 97.22 & 98.15 & 98.62 \\
\hline Reverse & & & 82.81 & 88.13 & 92.63 & 95.10 & 96.33 & 80.56 & 90.67 & 96.35 & 98.19 & 98.80 & 99.10 \\
\hline Random & & 74.65 & 86.21 & 94.37 & 97.21 & 98.14 & 98.61 & 74.84 & 86.84 & 94.77 & 97.41 & 98.28 & 98.71 \\
\hline ICBP & & 76.34 & 87.46 & 94.97 & 97.50 & 98.34 & 98.76 & 76.84 & 88.17 & 95.34 & 97.69 & 98.46 & 98.85 \\
\hline inCTPri & & 80.51 & 90.08 & 96.03 & 98.03 & 98.69 & 99.02 & 81.19 & 90.77 & 96.40 & 98.21 & 98.81 & 99.11 \\
\hline $\mathrm{ASPS}_{e}$ & & 80.76 & 90.27 & 96.11 & 98.07 & 98.72 & 99.04 & 80.39 & 90.41 & 96.25 & 98.14 & 98.76 & 99.07 \\
\hline $\mathrm{ASPS}_{r}$ & & 79.89 & 89.48 & 95.79 & 97.91 & 98.61 & 98.96 & 80.34 & 90.27 & 96.20 & 98.11 & 98.75 & 99.06 \\
\hline $\mathrm{ASPS}_{m}$ & & 81.45 & 90.65 & 96.26 & 98.15 & 98.77 & 99.08 & 81.41 & 90.88 & 96.44 & 98.23 & 98.82 & 99.12 \\
\hline
\end{tabular}


Table 9: The NAPFD metric (\%) for different prioritization techniques for subject program nametbl when executing the percentage of interaction test sequence.

\begin{tabular}{|c|c|c|c|c|c|c|c|c|c|c|c|c|c|}
\hline \multirow{2}{*}{ Method } & \multirow{2}{*}{ Strength } & \multicolumn{6}{|c|}{$p$ of ACTS Interaction Test Sequence Executed } & \multicolumn{6}{|c|}{$p$ of PICT Interaction Test Sequence Executed } \\
\hline & & $5 \%$ & $10 \%$ & $25 \%$ & $50 \%$ & $75 \%$ & $100 \%$ & $5 \%$ & $10 \%$ & $25 \%$ & $50 \%$ & $75 \%$ & $100 \%$ \\
\hline Original & \multirow{8}{*}{$\tau=2$} & 0 & 15.70 & 55.04 & 75.87 & 83.91 & 88.42 & 11.63 & 27.33 & 58.72 & 72.97 & 81.98 & $\overline{87.02}$ \\
\hline Reverse & & 37.21 & $\mathbf{5 7 . 5 6}$ & 81.78 & 90.02 & 93.35 & 95.21 & 11.63 & 23.84 & 61.43 & 79.36 & 86.24 & 90.09 \\
\hline Random & & 8.22 & 32.92 & 62.85 & 79.31 & 86.15 & 90.03 & 19.13 & 33.58 & 61.53 & 77.54 & 84.65 & 88.91 \\
\hline ICBP & & 19.15 & 34.49 & 68.01 & 83.55 & 89.04 & 92.11 & 18.92 & 33.98 & 65.27 & 80.48 & 86.90 & 90.57 \\
\hline inCTPri & & 19.15 & 34.49 & 68.01 & 83.55 & 89.04 & 92.11 & 18.92 & 33.98 & 65.27 & 80.48 & 86.90 & 90.57 \\
\hline $\mathrm{ASPS}_{e}$ & & 19.38 & 35.81 & 69.56 & 84.33 & 89.55 & 92.48 & 19.21 & 34.52 & 66.05 & 81.31 & 87.50 & 91.00 \\
\hline $\mathrm{ASPS}_{r}$ & & 18.21 & 34.21 & 67.28 & 83.06 & 88.71 & 91.87 & 19.23 & 34.35 & 64.69 & 80.26 & 86.72 & 90.44 \\
\hline $\mathrm{ASPS}_{m}$ & & 19.57 & 35.80 & 68.70 & 83.89 & 89.26 & 92.27 & 18.99 & 35.07 & 67.22 & 82.28 & 88.14 & 91.46 \\
\hline Original & \multirow{8}{*}{$\tau=3$} & 27.03 & 48.55 & 69.19 & 84.37 & 89.50 & 92.19 & 40.31 & 64.12 & 85.37 & 92.87 & 95.21 & 96.44 \\
\hline Reverse & & 72.38 & 82.99 & 91.63 & 95.77 & 97.16 & 97.89 & 18.60 & 53.16 & 82.19 & 91.32 & 94.17 & 95.66 \\
\hline Random & & .44 & 71.53 & 87.80 & 94.04 & 96.00 & 97.02 & 43.26 & 66.39 & 85.87 & 93.06 & 95.34 & 96.53 \\
\hline ICBP & & 53.77 & 72.93 & 88.91 & 94.59 & 96.36 & 97.29 & 46.24 & 69.31 & 88.11 & 94.21 & 96.10 & 97.10 \\
\hline inCTPri & & 60.26 & 78.46 & 91.32 & 95.77 & 97.16 & 97.88 & 48.86 & 71.82 & 89.26 & 94.77 & 96.48 & 97.38 \\
\hline $\mathrm{ASPS}_{e}$ & & 60.53 & 78.59 & 91.35 & 95.78 & 97.16 & 97.89 & 47.54 & 72.83 & 89.68 & 94.97 & 96.62 & 97.48 \\
\hline $\mathrm{ASPS}_{r}$ & & .32 & 76.43 & 90.37 & 95.30 & 96.84 & 97.65 & 47.21 & 71.03 & 88.35 & 94.25 & 96.14 & 97.13 \\
\hline $\mathrm{ASPS}_{m}$ & & 7.75 & 77.18 & 90.83 & 95.53 & 96.99 & 97.76 & 46.28 & 71.78 & 89.13 & 94.70 & 96.43 & 97.35 \\
\hline Original & \multirow{8}{*}{$\tau=4$} & 32.03 & 51.64 & 70.14 & 84.35 & 89.57 & 92.21 & 72.09 & 85.68 & 94.37 & 97.21 & 98.14 & $\overline{98.61}$ \\
\hline Reverse & & & & & & & & 74.84 & 86.63 & 94.75 & 97.40 & & 98.70 \\
\hline Random & & 7.58 & 88.29 & 95.39 & 97.69 & 98.46 & 98.85 & 78.14 & 88.57 & 95.49 & 97.76 & 98.50 & 98.88 \\
\hline ICBP & & 77.56 & 88.47 & 95.47 & 97.74 & 98.49 & 98.87 & 77.94 & 88.71 & 95.56 & 97.80 & 98.53 & 98.90 \\
\hline inCTPri & & 82.07 & 90.98 & 96.46 & 98.23 & 98.82 & 99.12 & 81.48 & 90.72 & 96.35 & 98.19 & 98.79 & 99.10 \\
\hline $\mathrm{ASPS}_{e}$ & & 83.20 & 91.56 & 96.68 & 98.34 & 98.89 & 99.17 & 82.51 & 91.21 & 96.55 & 98.29 & 98.86 & 99.14 \\
\hline $\mathrm{ASPS}_{r}$ & & 81.79 & 90.74 & 96.36 & 98.18 & 98.79 & 99.09 & 82.46 & 91.14 & 96.52 & 98.27 & 98.85 & 99.14 \\
\hline $\mathrm{ASPS}_{m}$ & & 82.92 & 91.40 & 96.62 & 98.31 & 98.87 & 99.16 & 82.40 & 91.14 & 96.52 & 98.28 & 98.85 & 99.14 \\
\hline Original & \multirow{8}{*}{$\tau=5$} & 32.19 & 52.79 & 70.47 & 84.59 & 89.71 & 92.30 & 88.85 & 94.55 & 97.81 & 98.91 & 99.27 & 99.45 \\
\hline Reverse & & 83.30 & 88.71 & 93.13 & 95.87 & 97.24 & 97.94 & 89.38 & 94.81 & 97.91 & 98.96 & 99.31 & 99.48 \\
\hline Random & & 88.02 & 94.10 & 97.63 & 98.82 & 99.21 & 99.41 & 87.77 & 93.99 & 97.58 & 98.80 & 99.20 & 99.40 \\
\hline ICBP & & 88.37 & 94.28 & 97.70 & 98.86 & 99.24 & 99.43 & 88.77 & 94.50 & 97.79 & 98.90 & 99.27 & 99.45 \\
\hline inCTPri & & 90.47 & 95.34 & 98.13 & 99.07 & 99.38 & 99.53 & 90.69 & 95.44 & 98.17 & 99.09 & 99.39 & 99.54 \\
\hline $\mathrm{ASPS}_{e}$ & & 91.11 & 95.66 & 98.25 & 99.13 & 99.42 & 99.57 & 91.16 & 95.68 & 98.26 & 99.14 & 99.42 & 99.57 \\
\hline $\mathrm{ASPS}_{r}$ & & 90.04 & 95.13 & 98.04 & 99.03 & 99.35 & 99.51 & 90.69 & 95.45 & 98.17 & 99.09 & 99.39 & 99.54 \\
\hline $\mathrm{ASPS}_{m}$ & & 90.97 & 95.59 & 98.23 & 99.12 & 99.41 & 99.56 & 90.73 & 95.47 & 98.18 & 99.09 & 99.39 & 99.55 \\
\hline
\end{tabular}

2) Does the ASPS method lead to higher fault detection 20 rates than intuitive prioritization methods such as reverse 21 prioritization and random prioritization? In this part, we 22 compare the ASPS method with Reverse and Random, in 23 terms of fault detection rate.

As shown in the tables, $\mathrm{ASPS}_{e}, \mathrm{ASPS}_{r}$, and $\mathrm{ASPS}_{m}$ have 25 higher NAPFD values than Reverse in $76.32 \%(145 / 190), 26$ $76.84 \%(146 / 190)$, and $78.42 \%(149 / 190)$ of cases, 27 respectively. Furthermore, these three ASPS methods 28 outperform Random in $97.37 \%$ (185/190), 95.79\% (182/190), 29 and $94.74 \%(180 / 190)$ of cases, respectively. Also, as the 30 values of $p$ increase, the improvement of the ASPS method ${ }_{31}$ over Reverse or Random decreases.

However, there are cases where either Reverse or Random ${ }_{33}$ obtain interaction test sequences with the highest NAPFD ${ }_{34}$ values. For instance, for subject program count with $\tau=4,35$ Reverse performs best among all prioritization strategies, ${ }_{36}$ regardless of $p$ value or interaction test suite construction tool ${ }_{37}$ (ACTS or PICT); likewise, for subject program flex with 38
ACTS at $\tau=2$, when $p=75 \%$ or $100 \%$, Random obtains interaction test sequences with the highest NAPFD values.

In conclusion, in $76.32 \% \sim 97.37 \%$ of cases, the ASPS method performs better than the two intuitive prioritization methods Reverse and Random, according to NAPFD. Furthermore, similar to Original, the ASPS method favors the cases where smaller percentages of interaction test sequence are executed, compared with Reverse and Random.

3) Is the ASPS method better than "fixed-strength prioritization" in terms of fault detection rates? In this part, we compare fault detection rates of interaction test sequences prioritized by the ASPS method against two implementations of "fixed-strength prioritization", ICBP and inCTPri.

For subject program flex at strength $\tau=2$, ICBP performs better than the ASPS method for some $p$ values; otherwise, the ASPS method has higher NAPFD values, regardless of $p$ value. More specifically, ASPS ASPS $_{r}$, and $\mathrm{ASPS}_{m}$ have higher rates of fault detection than ICBP in $85.79 \%(163 / 190)$, $71.58 \%(136 / 190)$, and $86.31 \%(164 / 190)$ of cases, 
respectively. The main reason for this phenomenon is that 20 ICBP is an implementation of "fixed-strength prioritization", 21 which means that each selected element is evaluated according 22 to a fixed strength $\tau$. In addition, ICBP does not actually 23 change the prioritization strength value throughout the 24 prioritization process, and may consequently detect faults with 25 the FTFI number $=\tau$ more quickly than ASPS, and faults with 26 the FTFI number $<\tau$ more slowly, because the ASPS method ${ }_{27}$ focuses on aggregate strengths.

According to the NAPFD values shown in tables, ASPS $_{e}, 29$ $\mathrm{ASPS}_{r}$, and $\mathrm{ASPS}_{m}$ have better performances than inCTPri, in 30 $70.00 \%$ (133/190), 43.16\% (82/190), and 70.53\% (134/190) 31 of cases, respectively. The improvements of the ASPS 32 methods over inCTPri in medium-sized programs (flex and ${ }_{33}$ grep) are larger than those in small-sized programs (count and ${ }_{34}$ nametbl). Additionally, as the values of $p$ increase, the 35 improvement of the ASPS method against inCTPri generally ${ }_{36}$ decreases. Similar to ICBP, inCTPri is an implementation of ${ }_{37}$ "fixed-strength prioritization", which means that it also 38 prioritizes each test case using a fixed strength. However, they have different performances, something which can be explained as follows: ICBP uses a fixed strength $\tau$ throughout the prioritization process, but inCTPri uses different strengths $(2,3, \cdots$, and $\tau)$ to guide the interaction test suite prioritization.

Both ICBP and inCTPri use only one strength to prioritize each combinatorial test case, while ASPS uses different strengths when choosing each, which could explain why it performs better in most cases. On the other hand, although neither ICBP nor inCTPri consider different strengths to guide the selection of each test case, the selected best candidate $t c$ also involves additional information on interaction coverage at higher strengths. For example, suppose that $t c$ covers a number of uncovered $\lambda$-wise value combinations, then according to the proof of Theorem 1, it can be concluded that $t c$ also covers a number of uncovered value combinations at strengths higher than $\lambda$. Furthermore, since the fault distribution of each subject program (the FTFI number) is unknown before testing, it is

Table 10: The NAPFD metric (\%) for different prioritization techniques for subject program flex when executing the percentage of interaction test sequence.

\begin{tabular}{|c|c|c|c|c|c|c|c|c|c|c|c|c|c|}
\hline \multirow{2}{*}{ Method } & \multirow{2}{*}{ Strength } & \multicolumn{6}{|c|}{$p$ of ACTS Interaction Test Sequence Executed } & \multicolumn{6}{|c|}{$p$ of PICT Interaction Test Sequence Executed } \\
\hline & & $5 \%$ & $10 \%$ & $25 \%$ & $50 \%$ & $75 \%$ & $100 \%$ & $5 \%$ & $10 \%$ & $25 \%$ & $50 \%$ & $75 \%$ & $100 \%$ \\
\hline Original & \multirow{8}{*}{$\tau=2$} & 5.88 & 12.50 & 34.56 & 44.11 & 55.88 & 64.27 & 8.82 & 16.91 & 53.92 & 72.40 & 77.63 & 81.28 \\
\hline Reverse & & 13.24 & 27.94 & 48.53 & 64.82 & 72.94 & 76.91 & 7.35 & 16.91 & 48.53 & 66.74 & 72.45 & 76.75 \\
\hline Random & & 5.68 & 27.39 & 51.69 & 67.61 & 74.18 & 77.69 & 15.03 & 27.48 & 53.25 & 69.58 & 75.82 & 79.87 \\
\hline ICBP & & 5.12 & 31.10 & 62.96 & 76.10 & 80.29 & 82.35 & 14.94 & 29.69 & 61.31 & 75.12 & 79.62 & 82.66 \\
\hline inCTPri & & 15.12 & 31.10 & 62.96 & 76.10 & 80.29 & 82.35 & 14.94 & 29.69 & 61.31 & 75.12 & 79.62 & 82.66 \\
\hline $\mathrm{ASPS}_{e}$ & & 16.41 & 32.28 & 63.19 & 75.96 & 80.19 & 82.27 & 16.59 & 28.25 & 60.11 & 74.82 & 79.60 & 82.67 \\
\hline $\mathrm{ASPS}_{r}$ & & 15.41 & 30.31 & 62.24 & 75.37 & 79.75 & 81.94 & 17.35 & 30.57 & 59.81 & 75.05 & 79.93 & 82.94 \\
\hline $\mathrm{ASPS}_{m}$ & & 15.44 & 31.32 & 62.60 & 75.73 & 80.05 & 82.17 & 14.41 & 29.26 & 61.29 & 75.31 & 79.79 & 82.77 \\
\hline Original & \multirow{8}{*}{$\tau=3$} & 26.96 & 45.83 & 66.82 & 77.27 & 81.00 & 83.96 & 22.55 & 46.81 & 72.61 & 80.42 & 83.03 & $\overline{85.00}$ \\
\hline Reverse & & .41 & 42.89 & 56.34 & 71.88 & 79.11 & 2.98 & 42.65 & 58.09 & 75.83 & 83.50 & 86.06 & 87.40 \\
\hline Random & & 5.35 & 52.77 & 72.59 & 81.51 & 84.92 & 87.10 & 36.17 & 51.43 & 71.02 & 79.99 & 83.36 & 85.29 \\
\hline ICBP & & 3.57 & 62.09 & 77.66 & 83.66 & 85.97 & 7.88 & 41.41 & 60.42 & 76.65 & 82.44 & 84.65 & 86.18 \\
\hline inCTPri & & 41.88 & 61.30 & 77.33 & 83.40 & 85.80 & 87.82 & 40.35 & 59.89 & 76.63 & 82.61 & 84.83 & 86.32 \\
\hline $\mathrm{ASPS}_{e}$ & & 44.54 & 62.55 & 77.61 & 83.62 & 5.92 & .84 & 38.26 & 60.39 & 77.43 & 82.84 & 85.04 & 86.52 \\
\hline $\mathrm{ASPS}_{r}$ & & 42.21 & 60.68 & 77.35 & 83.59 & 86.01 & 87.85 & 40.40 & 59.23 & 76.43 & 82.68 & 84.91 & 86.39 \\
\hline $\mathrm{ASPS}_{m}$ & & 43.50 & 62.33 & 77.96 & 83.91 & 86.18 & 88.10 & 42.09 & 61.44 & 77.21 & 82.77 & 84.81 & 86.23 \\
\hline Original & \multirow{8}{*}{$\tau=4$} & 2.11 & 51.70 & 68.20 & 78.28 & 83.79 & 87.90 & 41.67 & 49.88 & 72.43 & 80.33 & 83.79 & 86.43 \\
\hline Reverse & & .25 & 59.62 & 64.38 & 76.79 & 84.17 & 88.19 & 58.09 & 70.10 & 79.60 & 85.98 & 88.69 & 90.08 \\
\hline Random & & 51.75 & 68.36 & 81.31 & 87.77 & 90.74 & 92.85 & 52.52 & 67.10 & 80.16 & 85.49 & 87.88 & 89.36 \\
\hline ICBP & & 59.98 & 74.36 & 83.96 & 88.33 & 90.54 & 92.72 & 61.04 & 73.40 & 82.97 & 86.72 & 88.86 & 90.10 \\
\hline & & & & 84.01 & 88.32 & 90.66 & 92.82 & 61.82 & 74.21 & 83.37 & 86.93 & 88.67 & 89.83 \\
\hline $\operatorname{ASPS}_{e}$ & & 62.11 & 75.83 & 84.50 & 88.44 & 90.50 & 92.69 & 60.63 & 73.66 & 83.00 & 86.41 & 88.44 & 89.68 \\
\hline & & 59.90 & 73.92 & 83.48 & 88.36 & 90.72 & 92.80 & 59.25 & 72.25 & 82.41 & 86.50 & 88.51 & 89.78 \\
\hline $\mathrm{ASPS}_{m}$ & & 62.85 & 76.07 & 84.29 & 88.30 & 90.31 & 92.52 & 62.19 & 74.29 & 83.26 & 86.60 & 88.67 & 89.94 \\
\hline Original & \multirow{8}{*}{$\tau=5$} & 35.96 & 57.54 & 74.23 & 82.64 & 87.48 & 90.63 & 55.15 & 71.15 & 82.30 & 89.06 & 91.80 & 93.86 \\
\hline Reverse & & 52.81 & 60.55 & & 71.81 & 81.03 & 85.81 & 59.71 & 71.57 & 81.13 & 87.56 & 89.76 & 92.13 \\
\hline Random & & 65.23 & 77.11 & 86.13 & 90.77 & 93.09 & 94.72 & 61.14 & 74.23 & 84.26 & 89.01 & 91.52 & 93.36 \\
\hline ICBP & & 71.39 & 80.69 & 87.50 & 91.03 & 93.02 & 94.67 & 69.31 & 79.40 & 86.58 & 90.49 & 92.95 & 94.71 \\
\hline inCTPri & & 73.23 & 81.27 & 86.97 & 90.45 & 92.81 & 94.54 & 71.92 & 80.55 & 86.20 & 89.69 & 92.33 & 94.23 \\
\hline $\mathrm{ASPS}_{e}$ & & 73.36 & 81.71 & 87.54 & 91.02 & 93.09 & 94.76 & 70.84 & 80.52 & 86.98 & 90.59 & 93.05 & 94.77 \\
\hline & & 70.62 & 79.95 & 87.30 & 91.06 & 92.97 & 94.61 & 69.31 & 79.15 & 86.54 & 90.32 & 92.51 & 94.30 \\
\hline $\mathrm{ASPS}_{m}$ & & 73.91 & 81.73 & 87.02 & 90.48 & 92.74 & 94.44 & 72.34 & 80.69 & 86.53 & 90.27 & 92.66 & 94.49 \\
\hline
\end{tabular}


Table 11: The NAPFD metric $(\%)$ for different prioritization techniques for subject program grep when executing the percentage of interaction test sequence.

\begin{tabular}{|c|c|c|c|c|c|c|c|c|c|c|c|c|c|}
\hline \multirow{2}{*}{ Method } & \multirow{2}{*}{ Strength } & \multicolumn{6}{|c|}{$p$ of ACTS Interaction Test Sequence Executed } & \multicolumn{6}{|c|}{$p$ of PICT Interaction Test Sequence Executed } \\
\hline & & $5 \%$ & $10 \%$ & $25 \%$ & $50 \%$ & $75 \%$ & $100 \%$ & $5 \%$ & $10 \%$ & $25 \%$ & $50 \%$ & $75 \%$ & $100 \%$ \\
\hline Original & \multirow{8}{*}{$\tau=2$} & 25.00 & 32.50 & 37.27 & 41.96 & 51.18 & 60.54 & 25.00 & 32.50 & 41.82 & 66.74 & 74.71 & 78.62 \\
\hline Reverse & & 30.00 & 35.00 & 45.45 & 59.35 & 69.26 & 74.67 & 30.00 & 38.75 & 54.09 & 68.48 & 75.86 & 79.47 \\
\hline Random & & 33.03 & 44.30 & 60.15 & 70.35 & 75.34 & 78.75 & 32.78 & 45.09 & 62.85 & 74.23 & 79.33 & 82.04 \\
\hline ICBP & & 31.13 & 42.74 & 61.80 & 72.36 & 76.10 & 78.69 & 30.65 & 42.30 & 61.42 & 74.29 & 79.58 & 82.24 \\
\hline inCTPri & & 31.13 & 42.74 & 61.80 & 72.36 & 76.10 & 78.69 & 30.65 & 42.30 & 61.42 & 74.29 & 79.58 & 82.24 \\
\hline $\mathrm{ASPS}_{e}$ & & 36.55 & 49.55 & 66.35 & 75.35 & 78.66 & 80.90 & 31.05 & 44.35 & 64.70 & 76.23 & 80.89 & 83.22 \\
\hline $\mathrm{ASPS}_{r}$ & & 31.70 & 44.30 & 63.07 & 72.89 & 76.67 & 79.43 & 34.05 & 46.48 & 64.53 & 75.79 & 80.55 & 82.97 \\
\hline $\mathrm{ASPS}_{m}$ & & 33.15 & 46.25 & 64.97 & 74.03 & 77.57 & 80.05 & 33.45 & 45.73 & 64.94 & 76.39 & 80.99 & 83.29 \\
\hline Original & \multirow{8}{*}{$\tau=3$} & 37.86 & 44.33 & 61.58 & 76.84 & 84.56 & 88.50 & 50.00 & 62.00 & 77.43 & 86.00 & 90.63 & 93.00 \\
\hline Reverse & & 47.86 & 64.00 & 74.60 & 82.30 & 85.44 & 89.15 & 58.57 & 70.00 & 79.86 & 87.87 & 91.88 & 93.93 \\
\hline Random & & 57.17 & 67.65 & 78.22 & 84.87 & 88.47 & 90.99 & 58.11 & 69.50 & 80.58 & 87.28 & 90.93 & 93.18 \\
\hline ICBP & & 56.44 & 69.51 & 79.94 & 86.27 & 89.92 & 92.48 & 58.69 & 71.53 & 80.69 & 87.56 & 91.34 & 93.53 \\
\hline inCTPri & & 55.94 & 69.84 & 80.68 & 87.42 & 91.10 & 93.37 & 58.67 & 71.57 & 81.51 & 88.20 & 91.81 & 93.88 \\
\hline $\mathrm{ASPS}_{e}$ & & 59.30 & 71.21 & 80.81 & 86.65 & 90.45 & 92.88 & 58.33 & 72.07 & 82.67 & 88.88 & 92.36 & 94.29 \\
\hline $\mathrm{ASPS}_{r}$ & & 60.17 & 72.20 & 81.09 & 86.01 & 89.06 & 91.80 & 60.17 & 72.39 & 82.14 & 89.05 & 92.33 & 94.25 \\
\hline ASPS $_{m}$ & & 59.57 & 72.23 & 81.40 & 86.51 & 89.98 & 92.52 & 58.12 & 71.65 & 81.88 & 88.62 & 92.13 & 94.12 \\
\hline Original & \multirow{8}{*}{$\tau=4$} & 43.93 & 55.86 & 70.54 & 82.89 & 88.57 & 91.44 & 61.07 & 68.62 & 78.72 & 84.36 & 86.24 & 89.53 \\
\hline Reverse & & 63.93 & 67.24 & 75.88 & 82.99 & 86.05 & 89.56 & 51.79 & 64.31 & 79.93 & 89.80 & 93.20 & 94.90 \\
\hline Random & & 65.96 & 75.25 & 84.43 & 89.89 & 92.65 & 94.45 & 66.06 & 75.33 & 85.23 & 90.82 & 93.42 & 95.01 \\
\hline ICBP & & 67.97 & 77.58 & 86.44 & 92.38 & 94.91 & 96.19 & 70.94 & 78.59 & 86.22 & 92.36 & 94.90 & 96.18 \\
\hline inCTPri & & 71.02 & 79.43 & 87.07 & 92.85 & 95.22 & 96.42 & 71.22 & 79.44 & 87.74 & 93.13 & 95.42 & 96.57 \\
\hline $\mathrm{ASPS}_{e}$ & & 70.64 & 78.17 & 86.24 & 92.26 & 94.83 & 96.13 & 71.59 & 78.98 & 86.81 & 92.59 & 95.06 & 96.30 \\
\hline $\mathrm{ASPS}_{r}$ & & 71.44 & 79.62 & 87.94 & 93.39 & 95.58 & 96.69 & 70.09 & 79.74 & 87.62 & 92.91 & 95.28 & 96.46 \\
\hline $\mathrm{ASPS}_{m}$ & & 70.24 & 78.49 & 86.96 & 92.75 & 95.15 & 96.37 & 70.98 & 79.13 & 86.48 & 92.40 & 94.93 & 96.20 \\
\hline Original & \multirow{8}{*}{$\tau=5$} & 45.95 & 48.02 & 49.22 & 59.43 & 71.96 & 78.97 & 66.19 & 78.26 & 87.98 & 93.99 & 95.99 & 97.00 \\
\hline Reverse & & 64.29 & 72.33 & 77.11 & 86.10 & 90.73 & 93.05 & 79.76 & 85.00 & 94.04 & 97.02 & 98.01 & 98.51 \\
\hline Random & & 70.05 & 77.18 & 85.43 & 90.72 & 93.50 & 95.11 & 70.05 & 77.18 & 85.43 & 90.72 & 93.50 & 95.11 \\
\hline ICBP & & 72.32 & 80.33 & 88.88 & 94.36 & 96.24 & 97.18 & 72.32 & 80.33 & 88.88 & 94.36 & 96.24 & 97.18 \\
\hline inCTPri & & 74.00 & 81.41 & 88.95 & 94.44 & 96.29 & 97.22 & 74.52 & 82.47 & 90.57 & 95.28 & 96.85 & 97.64 \\
\hline $\mathrm{ASPS}_{e}$ & & 76.98 & 82.92 & 90.33 & 95.10 & 96.73 & 97.55 & 75.89 & 81.84 & 89.18 & 94.43 & 96.29 & 97.22 \\
\hline $\operatorname{ASPS}_{r}$ & & 76.60 & 82.61 & 89.07 & 94.16 & 96.10 & 97.08 & 75.30 & 81.78 & 89.52 & 94.50 & 96.34 & 97.25 \\
\hline $\operatorname{ASPS}_{m}$ & & 76.49 & 83.69 & 91.09 & 95.47 & 96.98 & 97.73 & 76.49 & 83.69 & 91.09 & 95.47 & 96.98 & 97.73 \\
\hline
\end{tabular}

reasonable that ICBP and inCTPri occasionally have better 20 fault detection rates than ASPS (especially $\mathrm{ASPS}_{r}$, as its 21 weighting distribution is assigned in a random manner). 22

In conclusion, according to the fault detection rates (the 23 NAPFD values), ASPS performs better than both 24 "fixed-strength prioritization" implementations, that is, ICBP 25 (in $71.58 \% \sim 86.32 \%$ of cases) and inCTPri (in about $70.00 \% 26$ of cases for $\mathrm{ASPS}_{e}$ and $\mathrm{ASPS}_{m}$; and in $43.16 \%$ of cases for 27 $\mathrm{ASPS}_{r}$ ).

4) Which weighting distribution for the ASPS method gives 29 the best fault detection rates? In this part, we study the fault ${ }_{30}$ detection rate of the ASPS method with three weighting ${ }_{31}$ distributions, so as to determine which weighting distribution 32 is best.

From the experimental data, no weighting distribution is 34 always best, because each weighting distribution performs best ${ }_{35}$ for some cases, but worst for others. Consider subject program ${ }_{36}$ grep (Table 8), for example: (a) ASPS with equal weighting ${ }_{37}$ distribution $\left(\mathrm{ASPS}_{e}\right)$ has the best NAPFD values for ACTS at strength $\tau=2$; (b) ASPS with random weighting distribution $\left(\mathrm{ASPS}_{r}\right.$ ) performs best for ACTS at strength $\tau=4$; and (c) ASPS with empirical FTFI percentage weighting distribution $\left(\mathrm{ASPS}_{m}\right.$ ) has the best fault detection at strength $\tau=5$, for both ACTS and PICT.

On the whole, however, equal and empirical FTFI percentage weighting distributions have better rates of fault detection than random weighting distributions. As shown in Fig. 2, $\operatorname{ASPS}_{e}$ achieves the best NAPFD values in $48.33 \%(91.83 / 190)$ of cases, followed by $\mathrm{ASPS}_{m}$ in $34.65 \%(65.83 / 190)$ of cases. In other words, for the ASPS method, equal and empirical FTFI percentage weighting distributions would be the better choice in practical testing.

In conclusion, although each weighting distribution can perform best in some cases, the equal and empirical FTFI percentage weighting distributions perform better than random weighting distribution.

5) Time-cost analysis: In this part, we further investigate 


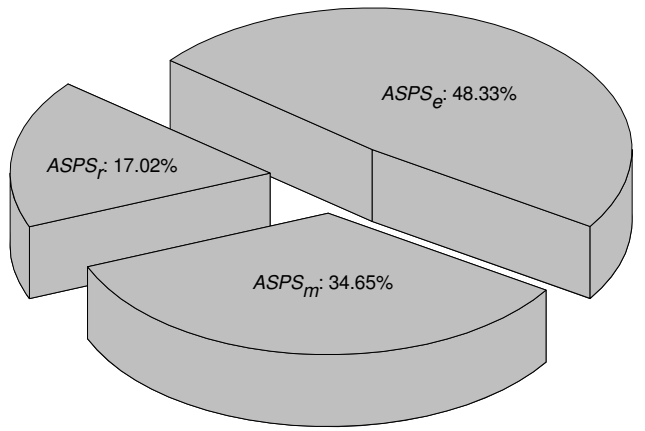

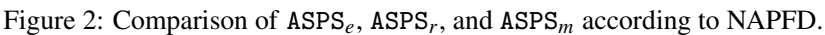

33

the time cost of the ICBP, inCTPri, and ASPS ${ }^{11}$ methods, to ${ }_{35}^{34}$ help guide practical use.

Table 12 presents the time cost (in seconds) for different prioritization techniques of interaction test suites on different ${ }^{36}$ subject programs. From the experimental data, we can observe ${ }^{37}$ that our method ASPS has very similar time cost to ICBP. It ${ }^{38}$ can also be observed that when the strength $\tau=2$, the time ${ }^{39}$ cost of ASPS is similar to that of inCTPri; but as the strength ${ }^{40}$ $\tau$ increases $(\tau=3,4,5)$, the time cost of ASPS differs from ${ }^{41}$ that of inCTPri. More specifically, for the two small-sized ${ }^{42}$ programs (count and nametbl), ASPS requires less time, but ${ }^{43}$ for the medium-sized programs (flex and grep), inCTPri has ${ }^{44}$ lower time costs.

According to the fault detection rates, ASPS generally has ${ }^{46}$ better performance than ICBP and inCTPri, especially when ${ }^{47}$ the percentage of interaction test sequence executed $(p)$ is ${ }^{48}$ lower, in which case, the prioritization time cost of ASPS is ${ }^{49}$ also lower. In other words, when testing resources are limited, ${ }^{50}$ ASPS should be chosen as the prioritization method; however, ${ }^{51}$ when testing resources are less constrained, inCTPri would ${ }^{52}$

${ }^{11}$ We do not consider Original, Reverse, and Random, because the cost of 55 these methods should be much less than that of the methods which make use of 56 some additional information (ICBP, inCTPri, and ASPS). Furthermore, since the three implementations of ASPS $\left(\mathrm{ASPS}_{e}, \mathrm{ASPS}_{r}\right.$, and $\left.\mathrm{ASPS}_{m}\right)$ have similar ${ }^{57}$ prioritization time, we use ASPS to represent all three. be a better choice because it requires less prioritization time for medium-sized programs.

6) Conclusion: The experimental study using real programs shows that although each method may sometimes obtain the highest NAPFD values, the ASPS method (regardless of $\mathrm{ASPS}_{e}, \mathrm{ASPS}_{r}$, and $\mathrm{ASPS}_{m}$ ) performs better than Original, Reverse, Random, and ICBP in at least $70 \%$ of cases. Furthermore, the ASPS method (except for $\mathrm{ASPS}_{r}$ ) also has better fault detection rates than inCTPri in about $70 \%$ of cases. Additionally, equal and empirical FTFI percentage weighting distributions give better performance for ASPS than random weighting distribution. With regard to the time cost for different prioritization strategies, the ASPS method has similar performance to ICBP, and higher costs than inCTPri for some subject programs but lower for others.

\subsection{Threats to validity}

In spite of our best efforts, our experiments may face three threats to validity:

(1) The experimental setup: The experimental programs are well-coded and tested. We have tried to manually cross-validate our analyzed programs on small examples, and we are confident of the correctness of the experimental and simulation set-up.

(2) The selection of experimental data: Two commonly-used tools (ACTS and PICT) were used for generating different covering arrays at different strength values. Although they are used in the field of combinatorial interaction testing, both of them use greedy algorithms. Only four test profiles were used for the simulations, which, although representative, were limited. For the real-life experiments, we examined only four subject programs, two of which were of a relatively small size. To address these potential threats, additional studies will be conducted in the future using many other test profiles, a greater number of subject programs, and different algorithms for covering array construction such as simulated annealing based algorithms $[8,15]$.

(3) The evaluation of experimental results: In order to objectively evaluate the effectiveness of the proposed method,

Table 12: Time comparisons (in seconds) of different prioritization techniques of interaction test suites.

\begin{tabular}{|c|c|c|c|c|c|c|c|c|c|}
\hline \multirow{2}{*}{ Subject Program } & \multirow{2}{*}{ Prioritization Strategy } & \multicolumn{4}{|c|}{ ACTS } & \multicolumn{4}{|c|}{ PICT } \\
\hline & & $\tau=2$ & $\tau=3$ & $\tau=4$ & $\tau=5$ & $\tau=2$ & $\tau=3$ & $\tau=4$ & $\tau=5$ \\
\hline \multirow{3}{*}{ count } & ICBP & 0.04 & 0.25 & 1.30 & 3.11 & 0.04 & 0.26 & 1.47 & 3.49 \\
\hline & inCTPri & 0.04 & 0.22 & 1.34 & 5.48 & 0.04 & 0.24 & 1.59 & 6.15 \\
\hline & ASPS & 0.04 & 0.25 & 1.30 & 3.12 & 0.04 & 0.26 & 1.47 & 3.51 \\
\hline \multirow{3}{*}{ nametbl } & ICBP & 0.07 & 0.49 & 2.27 & 3.65 & 0.07 & 0.44 & 2.35 & 3.65 \\
\hline & inCTPri & 0.07 & 0.45 & 2.74 & 8.36 & 0.07 & 0.45 & 2.88 & 8.71 \\
\hline & ASPS & 0.07 & 0.49 & 2.27 & 3.66 & 0.07 & 0.44 & 2.35 & 3.66 \\
\hline \multirow{3}{*}{ flex } & ICBP & 0.13 & 1.57 & 10.42 & 38.64 & 0.12 & 1.50 & 10.72 & 34.61 \\
\hline & inCTPri & 0.13 & 1.00 & 5.86 & 27.10 & 0.12 & 1.11 & 6.55 & 22.26 \\
\hline & ASPS & 0.13 & 1.57 & 10.53 & 38.94 & 0.12 & 1.50 & 10.81 & 34.97 \\
\hline \multirow{3}{*}{ grep } & ICBP & 0.31 & 8.13 & 57.63 & 190.59 & 0.37 & 7.89 & 54.85 & 195.70 \\
\hline & inCTPri & 0.31 & 5.54 & 31.80 & 92.27 & 0.37 & 5.25 & 32.81 & 99.66 \\
\hline & ASPS & 0.31 & 8.19 & 58.32 & 191.87 & 0.37 & 7.89 & 55.40 & 196.94 \\
\hline
\end{tabular}


the covering value combinations and the fault detection were 56 measured with the APCC and NAPFD metrics, which are 57 commonly used in the study of test case prioritization. 58 Additionally, we only presented the averages for each 59 prioritization strategy rather than a full statistical analysis, which is something we will prepare in the future.

\section{Conclusion and Future Work}

Combinatorial interaction testing [29] is a well-accepted ${ }_{63}^{62}$ testing technique, but due to often limited testing resources, ${ }_{64}$ prioritization of interaction test suites in combinatorial ${ }_{65}^{64}$ interaction testing has become very important. A dissimilarity ${ }_{66}$ 12 measure to evaluate combinatorial test cases is introduced in ${ }_{67}$ 13 this paper, based on which, a new pure prioritization strategy ${ }_{68}$ 14 for interaction test suites is proposed, "aggregate-strength ${ }_{69}$ 15 prioritization". Compared with traditional interaction coverage based test prioritization ("fixed-strength prioritization") $[1,2,4-7,18,30-33,37,39,40]$, the proposed method uses more information to guide prioritization of test ${ }_{73}$ 19 suites. From the perspective of covering value combinations ${ }_{74}$ 20 and fault detection, experimental results demonstrate that in most cases our method outperforms the test-case-generation 22 prioritization, the reverse test-case-generation prioritization, ${ }^{75}$ and the random prioritization. Additionally, in most cases, our 24 method has better performance than two implementations of 77 25 "fixed-strength prioritization" while maintaining a similar time ${ }^{78}$ cost.

Similar to "fixed-strength prioritization", our prioritization ${ }_{81}^{80}$ strategy is not limited to conventional software. For example, 82 event-driven software is a widely used category of software ${ }^{83}$ that takes sequences of events as input, alters state, and outputs ${ }_{85}^{84}$ new event sequences $[4,5]$. It would be interesting to apply ${ }_{86}^{85}$ our strategy to different software including event-driven 87 software in the future. Additionally, since the challenges of 88 which weighting to be used and of whether to use ${ }_{90}^{89}$ fixed-strength or aggregate-strength prioritization strategy may ${ }_{91}^{90}$ depend on characteristics of the system under test, it would be 92 useful, but challenging, to investigate the application scope of ${ }^{93}$ each prioritization strategy (including different weighting ${ }_{95}^{94}$ distributions).

The interaction test suite construction tool in our study, 97 PICT [10], uses a greedy algorithm to generate (select) ${ }^{98}$ combinatorial test cases: it selects an element as the next test ${ }_{100}^{99}$ cases such that it covers the largest number of value ${ }_{101}$ combinations at a given strength $\tau$ (the largest $U V C D_{\tau}$ ). Since ${ }^{102}$ PICT considers $U V C D_{\tau}$ as the benefit for each combinatorial ${ }^{103}$ test case, PICT actually considers the prioritization during it ${ }_{105}^{104}$ the process of combinatorial test case generation. According to 106 Qu's classification [32], therefore, PICT belongs to the ${ }^{107}$ category of re-generation prioritization. Although the ${ }^{108}$ simulation results (Section 5.1) indicate that the differences ${ }_{110}^{109}$ between PICT and other prioritization methods are small in 111 terms of the APCC metric, the experimental results against ${ }^{112}$ real-life programs (Section 5.2) show that other prioritization ${ }_{114}^{113}$ methods can obtain higher fault detection rates than PICT $_{115}^{114}$ 55 according to the NAPFD metric. In the future, it will be ${ }_{116}$ important and interesting for us to solve the problem: do interaction test sequences obtained by re-generation prioritization need to be further ordered by the pure prioritization category?

\section{Acknowledgements}

The authors would like to thank Christopher M. Lott for providing us the source code and failure reports for count and nametbl, and the Software-artifact Infrastructure Repository (SIR) [11] for providing the source code and fault data for flex and grep. We would also like to thank D. Richard Kuhn for providing us the ACTS tool, and Justyna Petke for helping in the experimental setup. Additionally, we would like to acknowledge T. Y. Chen for the many helpful discussions and comments. This work is partly supported by the National Natural Science Foundation of China (Grant No. 61202110), the Natural Science Foundation of Jiangsu Province (Grant No. BK2012284), and the Senior Personnel Scientific Research Foundation of Jiangsu University (Grant No. 14JDG039).

\section{References}

[1] Bryce, R. C., Colbourn, C. J., 2005. Test prioritization for pairwise interaction coverage. In: Proceedings of the 1st International Workshop on Advances in Model-based Testing (A-MOST'05). pp. 1-7.

[2] Bryce, R. C., Colbourn, C. J., 2006. Prioritized interaction testing for pairwise coverage with seeding and contraints. Information and Software Technology 48 (10), 960-970.

[3] Bryce, R. C., Colbourn, C. J., Cohen, M. B., 2005. A framework of greedy methods for constructing interaction test suites. In: Proceedings of the 27th International Conference on Software Engineering (ICSE'05). pp. 146-155.

[4] Bryce, R. C., Memon, A. M., 2007. Test suite prioritization by interaction coverage. In: Proceedings of the Workshop on Domain Specific Approaches to Software Test Automation (DoSTA'07). pp. 1-7.

[5] Bryce, R. C., Sampath, S., Memon, A. M., 2011. Developing a single model and test prioritization strategies for event-driven software. IEEE Transactions on Software Engineering 37 (1), 48-64.

[6] Bryce, R. C., Sampath, S., Pedersen, J. B., Manchester, S., 2011. Test suite prioritization by cost-based combinatorial interaction coverage. International Journal of Systems Assurance Engineering and Management 2 (2), 126-134.

[7] Chen, X., Gu, Q., Zhang, X., Chen, D., 2009. Building prioritized pairwise interaction test suites with ant colony optimization. In: Proceedings of the 9th International Conference on Quality Software (QSIC'09). pp. 347-352.

[8] Cohen, M. B., Gibbons, P. B., Mugridge, W. B., Colbourn, C. J., 2003. Constructing test suites for interaction testing. In: Proceedings of the 25th International Conference on Software Engineering (ICSE '03). pp. 38-48.

[9] Cohen, M. B., Gibbons, P. B., Mugridge, W. B., Colbourn, C. J., Collofello, J. S., 2003. Variable strength interaction testing of components. In: Proceedings of the 27th Annual International Conference on Computer Software and Applications (COMPSAC'03). pp. 413-418.

[10] Czerwonka, J., 2006. Pairwise testing in real world: Practical extensions to test case generators. In: Proceedings of the 24th Pacific Northwest Software Quality Conference (PNSQC'06). pp. 419-430.

[11] Do, H., Elbaum, S. G., Rothermel, G., 2005. Supporting controlled experimentation with testing techniques: An infrastructure and its potential impact. Empirical Software Engineering 10 (4), 405-435.

[12] Elbaum, S., Malishevsky, A., Rothermel, G., 2001. Incorporating varying test costs and fault severities into test case prioritization. In: Proceedings of the 23rd International Conference on Software Engineering (ICSE'01). pp. 329-338. 
[13] Elbaum, S., Malishevsky, A. G., Rothermel, G., 2002. Test case 72 prioritization: A family of empirical studies. IEEE Transaction on 73 Software Engineering 28 (2), 159-182.

[14] Fouché, S., Cohen, M. B., Porter, A., 2009. Incremental covering array 75 failure characterization in large configuration spaces. In: Proceedings 76 of the 18th International Symposium on Software Testing and Analysis 77 (ISSTA'09). pp. 177-188.

[15] Garvin, B. J., Cohen, M. B., Dwyer, M. B., February 2011. Evaluating 79 improvements to a meta-heuristic search for constrained interaction 80 testing. Empirical Software Engineering 16 (1), 61-102.

[16] Ghandehari, L. S. G., Lei, Y., Xie, T., Kuhn, R., Kacker, R., 2012. 82 Identifying failure-inducing combinations in a combinatorial test set. In: 83 Proceedings of the 5th International Conference on Software Testing, 84 Verification and Validation (ICST'12). pp. 370-379.

[17] Grindal, M., Lindström, B., Offutt, J., Andler, S. F., 2006. An evaluation 8 of combination strategies for test case selection. Empirical Software 87 Engineering 11 (4), 583-611.

[18] Huang, R., Chen, J., Li, Z., Wang, R., Lu, Y., 2014. Adaptive random 89 prioritization for interaction test suites. In: Proceedings of the 29th 90 Symposium On Applied Computing (SAC'14), To appear.

[19] Huang, R., Chen, J., Zhang, T., Wang, R., Lu, Y., 2013. Prioritizing 92 variable-strength covering array. In: Proceedings of the IEEE 37th Annual 93 Computer Software and Applications Conference (COMPSAC'13). pp. 94 502-511.

[20] Huang, R., Xie, X., Chen, T. Y., Lu, Y., 2012. Adaptive random 96 test case generation for combinatorial testing. In: Proceedings of the 97 IEEE 36th Annual Computer Software and Applications Conference 98 (COMPSAC'12). pp. 52-61.

[21] Huang, Y.-C., Peng, K.-L., Huang, C.-Y., 2012. A history-based cost-100 cognizant test case prioritization technique in regression testing. Journal 101 of Systems and Software 85 (3), 626-637.

[22] Jiang, B., Zhang, Z., Chan, W. K., Tse, T. H., 2009. Adaptive random test 103 case prioritization. In: Proceedings of the 24th IEEE/ACM International 104 Conference on Automated Software Engineering (ASE'09). pp. 233-244.105

[23] Kuhn, D. R., Reilly, M. J., 2002. An investigation of the applicability of 106 design of experiments to software testing. In: Proceedings of the 27th 107 Annual NASA Goddard/IEEE Software Engineering Workshop (SEW27’02). pp. 91-95.

[24] Kuhn, D. R., Wallace, D. R., Gallo, A. M., 2004. Software fault interactions and implications for software testing. IEEE Transaction on Software Engineering 30 (6), 418-421.

[25] Lei, Y., Kacker, R., Kuhn, D. R., Okun, V., 2008. Ipog/ipod: Efficient test generation for multi-way software testing. Software Testing, Verification, and Reliability 18 (3), 125-148.

[26] Li, Z., Harman, M., Hierons, R., 2007. Search algorithms for regression test case prioritization. IEEE Transactions on Software Engineering 33 (4), 225-237.

[27] Lott, C., Rombach, H., 1996. Repeatable software engineering experiments for comparing defect-detection techniques. Empirical Software Engineering 1 (3), 241-277.

[28] Mei, L., Chan, W. K., Tse, T. H., Merkel, R. G., 2011. Xml-manipulating test case prioritization for xml-manipulating services. Journal of Systems and Software 84 (4), 603-619.

[29] Nie, C., Leung, H., 2011. A survey of combinatorial testing. ACM Computer Survey 43 (2), 11:1-11:29.

[30] Petke, J., Yoo, S., Cohen, M. B., Harman, M., 2013. Efficiency and early fault detection with lower and higher strength combinatorial interaction testing. In: Proceedings of the 12th Joint Meeting on European Software Engineering Conference and the ACM SIGSOFT Symposium on the Foundations of Software Engineering (ESEC/FSE'13). pp. 26-36.

[31] Qu, X., Cohen, M. B., Rothermel, G., 2008. Configuration-aware regression testing: an empirical study of sampling and prioritization. In: Proceedings of the 17th International Symposium on Software Testing and Analysis (ISSTA'08). pp. 75-86

[32] Qu, X., Cohen, M. B., Woolf, K. M., 2007. Combinatorial interaction regression testing: A study of test case generation and prioritization. In: Proceedings of the IEEE International Conference on Software Maintenance (ICSM'07). pp. 255-264.

[33] Qu, X., Cohen, M. B., Woolf, K. M., 2013. A study in prioritization for higher strength combinatorial testing. In: Proceedings of the 2nd International Workshop on Combinatorial Testing, (IWCT'13). pp. 285-
294.

[34] Rothermel, G., Untch, R. H., Chu, C., Harrold, M. J., 2001. Prioritizing test cases for regression testing. IEEE Transactions on Software Engineering 27 (10), 929-948.

[35] Seroussi, G., Bshouty, N. H., 1988. Vector sets for exhaustive testing of logic circuits. IEEE Transactions on Information Theory 34 (3), 513-522.

[36] Srikanth, H., Banerjee, S., 2012. Improving test efficiency through system test prioritization. Journal of Systems and Software 85 (5), 1176-1187.

[37] Srikanth, H., Cohen, M. B., Qu, X., 2009. Reducing field failures in system configurable software: Cost-based prioritization. In: Proceedings of the 20th International Symposium on Software Reliability Engineering (ISSRE'09). pp. 61-70.

[38] Tai, K. C., Lei, Y., 2002. A test generation strategy for pairwise testing. IEEE Transaction on Software Engineering 28 (1), 109-111.

[39] Wang, Z., 2009. Test case generation and prioritization for combinatorial testing. Ph.D. thesis, Southeast University, Nanjing, Jiangsu, China.

[40] Wang, Z., Chen, L., Xu, B., Huang, Y., 2011. Cost-cognizant combinatorial test case prioritization. International Journal of Software Engineering and Knowledge Engineering 21 (6), 829-854.

[41] Wong, W. E., Horgan, J. R., London, S., Bellcore, H. A., 1997. A study of effective regression testing in practice. In: Proceedings of the 8th International Symposium on Software Reliability Engineering (ISSRE'97). pp. 264-274.

[42] Yilmaz, C., Cohen, M. B., Porter, A. A., 2006. Covering arrays for efficient fault characterization in complex configuration spaces. IEEE Transactions on Software Engineering 32 (1), 20-34.

[43] Yoon, H., Choi, B., 2011. A test case prioritization based on degree of risk exposure and its empirical study. International Journal of Software Engineering and Knowledge Engineering 21 (02), 191-209.

[44] Zhang, L., Hou, S.-S., Guo, C., Xie, T., Mei, H., 2009. Time-aware test-case prioritization using integer linear programming. In: Proceedings of the 18th International Symposium on Software Testing and Analysis (ISSTA'09). pp. 213-224.

[45] Zhang, Z., Zhang, J., 2011. Characterizing failure-causing parameter interactions by adaptive testing. In: Proceedings of the 20th International Symposium on Software Testing and Analysis (ISSTA'11). pp. 331-341. 